\title{
A Checklist of the Aquatic Invertebrates of the Delaware River Basin, 1990-2000
}

By Michael D. Bilger, Karen Riva-Murray, and Gretchen L. Wall

Data Series 116

U.S. Department of the Interior

U.S. Geological Survey 


\title{
U.S. Department of the Interior Gale A. Norton, Secretary
}

\author{
U.S. Geological Survey \\ Charles G. Groat, Director
}

\section{U.S. Geological Survey, Reston, Virginia: 2005}

\author{
For sale by U.S. Geological Survey, Information Services \\ Box 25286, Denver Federal Center \\ Denver, CO 80225 \\ For more information about the USGS and its products: \\ Telephone: 1-888-ASK-USGS \\ World Wide Web: http://www.usgs.gov/
}

Any use of trade, product, or firm names in this publication is for descriptive purposes only and does not imply endorsement by the U.S. Government.

Although this report is in the public domain, permission must be secured from the individual copyright owners to reproduce any copyrighted materials contained within this report.

Suggested citation:

Bilger, M.D., Riva-Murray, Karen, and Wall, G.L., 2005, A checklist of the aquatic invertebrates of the Delaware River Basin, 1990-2000: U.S. Geological Survey Data Series 116, 29 p. 


\section{FOREWORD}

The U.S. Geological Survey (USGS) is committed to providing the Nation with accurate and timely scientific information that helps enhance and protect the overall quality of life and that facilitates effective management of water, biological, energy, and mineral resources (http://www. usgs.gov/). Information on the quality of the Nation's water resources is critical to assuring the long-term availability of water that is safe for drinking and recreation and suitable for industry, irrigation, and habitat for fish and wildlife. Population growth and increasing demands for multiple water uses make water availability, now measured in terms of quantity and quality, even more essential to the long-term sustainability of our communities and ecosystems.

The USGS implemented the National Water-Quality Assessment (NAWQA) Program in 1991 to support national, regional, and local information needs and decisions related to water-quality management and policy (http://water.usgs.gov/nawqa). Shaped by and coordinated with ongoing efforts of other

Federal, State, and local agencies, the NAWQA Program is designed to answer: What is the condition of our Nation's streams and ground water? How are the conditions changing over time? How do natural features and human activities affect the quality of streams and ground water, and where are those effects most pronounced? By combining information on water chemistry, physical characteristics, stream habitat, and aquatic life, the NAWQA Program aims to provide science-based insights for current and emerging water issues and priorities.

From 1991-2001, the NAWQA Program completed interdisciplinary assessments in 51 of the Nation's major river basins and aquifer systems, referred to as Study Units (http://water.usgs.gov/nawqa/ studyu.htm/. Baseline conditions were established for comparison to future assessments, and longterm monitoring was initiated in many of the basins. During the next decade, 42 of the 51 Study Units will be reassessed so that 10 years of comparable monitoring data will be available to determine trends at many of the Nation's streams and aquifers. The next 10 years of study also will fill in critical gaps in characterizing water-quality conditions, enhance understanding of factors that affect water quality, and establish links between sources of contaminants, the transport of those contaminants through the hydrologic system, and the potential effects of contaminants on humans and aquatic ecosystems.

The USGS aims to disseminate credible, timely, and relevant science information to inform practical and effective water-resource management and strategies that protect and restore water quality. We hope this NAWQA publication will provide you with insights and information to meet your needs, and will foster increased citizen awareness and involvement in the protection and restoration of our Nation's waters.

The USGS recognizes that a national assessment by a single program cannot address all waterresource issues of interest. External coordination at all levels is critical for a fully integrated understanding of watersheds and for cost-effective management, regulation, and conservation of our Nation's water resources. The NAWQA Program, therefore, depends on advice and information from other agencies - Federal, State, interstate, Tribal, and local—as well as nongovernmental organizations, industry, academia, and other stakeholder groups. Your assistance and suggestions are greatly appreciated.

Robert M. Hirsch

Associate Director for Water 


\section{Contents}

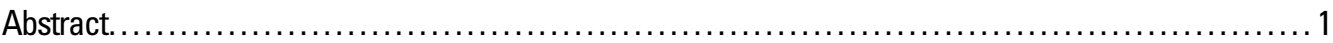

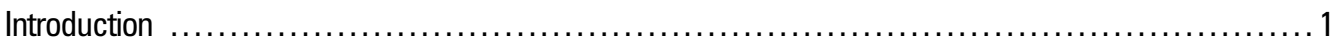

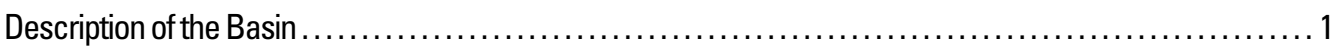

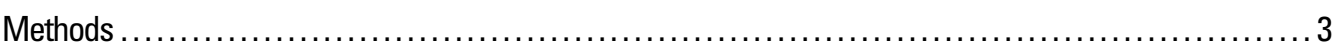

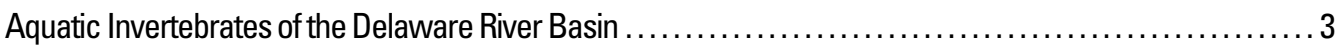

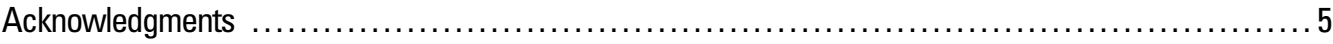

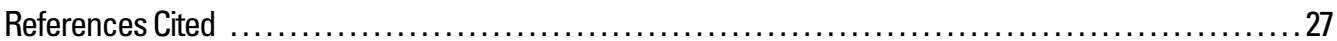

\section{Figures}

1. Map showing ecoregions and locations and types of sampling sites within the

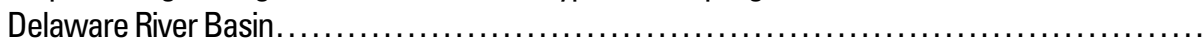

\section{Tables}

1. Agencies and programs from which benthic-invertebrate data were obtained for checklist of taxa in the Delaware River Basin .4

2. Checklist of the aquatic invertebrates of the Delaware River Basin, $1990-2000 \ldots \ldots \ldots \ldots \ldots \ldots 6$ 


\section{Conversion Factors and Datum}

\begin{tabular}{ccl}
\hline Multiply & By & To obtain \\
\hline & & \\
\hline Area & \\
square kilometer $\left(\mathrm{km}^{2}\right)$ & 0.3861 & square mile $\left(\mathrm{mi}^{2}\right)$ \\
\hline
\end{tabular}




\title{
A Checklist of the Aquatic Invertebrates of the Delaware River Basin, 1990-2000
}

\author{
By Michael D. Bilger, Karen Riva-Murray, and Gretchen L. Wall
}

\section{Abstract}

This paper details a compilation of aquatic-invertebrate taxa collected at 1,080 sites as part of 13 surface-water-quality studies completed by selected Federal, state, and local environmental agencies during 1990-2000, within the $32,893-\mathrm{km}^{2}$ area of the Delaware River Basin. This checklist is intended to be a "working list" of aquatic invertebrates that can be applied successfully to the calculation and interpretation of various biological estimators to determine the status of water quality and can be used as a foundation to document the current state of biodiversity. It is not intended as a comprehensive historical inventory of the literature or of private and public holdings. A total of 11 phyla comprising 20 classes, 46 orders, 196 families, 685 genera, and 835 species were recorded.

\section{Introduction}

Since the acceptance of the Saprobien system of Kolkwitz and Marsson (1908; 1909), the work of pioneering benthic scientists such as H.B. Noel Hynes (1960; 1970), and later researchers like Plafkin and others (1989), it was recognized that benthic-invertebrate assemblages were measurable indicators of localized conditions because of their complex life cycles of a year or more, their mostly sedentary nature, and their ability to react in a predictable way to human influences on aquatic ecosystems. These earlier efforts to document water-quality effects dealt mostly with organic pollutants. As the science has progressed, benthic assessments have evolved into multi-disciplined approaches that remain faunistic but also include habitat and geomorphic analyses of lotic systems. Newer efforts are concerned with understanding the native fauna and managing waters for maximized biological quality, including taking corrective action when monitoring indicates trouble (Cairns and Pratt, 1993). Barbour and others (1999) contains both multimetric and multivariate statistical methods for determining ecosystem effects on benthic-invertebrate communities. Accurate regional taxa lists and their accompanying pollution tolerance values are important to these assessments and interpretations and will gain increasing importance in the establishment of nar- rative or numeric regulatory values for biologically based criteria (U.S. Environmental Protection Agency, 1994).

After the works of E.O. Wilson (1984; 1988; 2000), the subject of biodiversity has been identified to represent the basic fabric of life. Because biodiversity is in a rapidly diminishing phase, it is increasingly difficult to provide a full accountability of what sorts of organisms were present before human influences eliminated them. Even within the fauna of North America, it is estimated that less than 50 percent of the arthropod biodiversity has been described (Kosztarab and Schaefer, 1990; Rawlins and Bier, 1998). Taxonomic studies can record and describe new taxa, determine the phylogenetic relation and taxonomic status of various taxa, provide a sound classification, and produce taxonomic keys and other tools to aid in accurate identification (K.C. Kim, State College, Pa., unpubl. data, 2001). Lists of taxa within the Delaware River Basin, as presented in this paper, will aid in the baseline effort to explain the status of the aquatic-invertebrate biodiversity even at the inconsistent level of taxonomic identification and assessment.

\section{Description of the Basin}

The non-tidal Delaware River Basin encompasses more than $32,893 \mathrm{~km}^{2}$ (fig. 1) and includes parts of the States of Pennsylvania, $\left(16,744 \mathrm{~km}^{2}\right)$, New Jersey $\left(7,690 \mathrm{~km}^{2}\right)$, New York $\left(6,120 \mathrm{~km}^{2}\right)$, and Delaware (2,507 km²) (Fischer, 2004). The headwaters of the Delaware River begin in the Catskill Mountains in the northern part of the basin. The river becomes tidally influenced in the area of Trenton, N.J., and is saline just south of Philadelphia, Pa. Two major tributaries of the Delaware River are the Lehigh $\left(4,903 \mathrm{~km}^{2}\right)$ and Schuylkill Rivers $\left(3,520 \mathrm{~km}^{2}\right)$. There are also a number of large reservoirs within the basin, including three operated by the City of New York for water supply and other uses.

The Delaware River Basin covers parts of eight ecoregions (Omernick, 1987; U.S. Environmental Protection Agency, 1999): the Northeastern Highlands, Northern Appalachian Plateau and Uplands, North Central Appalachians, Middle Atlantic Coastal Plain, Northern Piedmont, Southeastern Plains, Ridge and Valley, and Atlantic Coastal Pine Barrens. A wide range of 


\section{EXPLANATION}

$\triangle \quad$ COUNTY

- DELAWARE RIVER BASIN COMMISSION

- STATE

$\checkmark$ U.S. ENVIRONMENTAL PROTECTION AGENCY ENVIRONMENTAL MONITORING AND ASSESSMENT PROGRAM

$\nabla$ U.S. ENVIRONMENTAL PROTECTION AGENCY - REGION III

- U.S. GEOLOGICAL SURVEY, BIOLOGICAL RESOURCES DISCIPLINE

- U.S. GEOLOGICAL SURVEY, WATER RESOURCES DISCIPLINE

\section{ECOREGIONS}

NORTHEASTERN HIGHLANDS

NORTHERN APPALACHIAN PLATEAU AND UPLANDS

NORTH CENTRAL APPALACHIANS

MIDDLE ATLANTIC COASTAL PLAIN

NORTHERN PIEDMONT

SOUTHEASTERN PLAINS

RIDGE AND VALLEY

ATLANTIC COASTAL PINE BARRENS

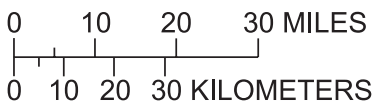

.


topography describes the basin from nearly flat in the coastal area, to rolling hills and broad uplands, to ridges and valleys, to glaciated plateaus. Altitude varies from sea level in the south to more than 1,000 meters in the north. According to 1992 landuse data, about 60 percent of the basin is forested, 24 percent agricultural, 9 percent urban, and 7 percent surface-water bodies and other miscellaneous uses (Vogelmann and others, 1998). Most of the population and urban land use is along the Delaware Estuary. Although the population within the basin has not increased significantly over the past 20 years, there has been a pattern of intense "urban flight" from the larger cities, creating an expansive suburbia.

\section{Methods}

Data were collected and compiled from Federal, state, and local environmental agencies active within the boundaries of the Delaware River Basin as defined by the U.S. Geological Survey (table 1). These agencies were selected on the basis of their common biomonitoring approach to document conditions and changes in surface-water quality from lotic habitats by using benthic-invertebrate community measures over roughly the past decade. Field-collection techniques were not standardized; only those data resulting from laboratory identifications were included (field-identified results from the Pennsylvania Department of Environmental Protection Unassessed Waters Program were not used). Historical records from extensive literature reviews and the examination of public and private specimen holdings were considered beyond the scope of this paper, as were data from distributional surveys of particular macroinvertebrate groups. It is hoped that this checklist be expanded in scope as a future endeavor and combined with other existing taxa lists such as those for Trichoptera (Masteller and others, 2004; Masteller and Flint, 1992), Plecoptera (Masteller and others, 2004; J. Earle, Harrisburg, Pa., unpub. data, 2003), Ephemeroptera (G. Hoover, State College, Pa., unpub. data, 2003), Simuliidae (Adler and Kim, 1986; D. Rebuck, Harrisburg, Pa., unpub. data, 2003), freshwater mussels (Strayer and Jirka, 1997; Lellis, 2001), Culicidae (M. Hutchinson, Harrisburg, Pa., unpub. data, 2003), Tabanidae (F. Fees, State College, Pa., unpub. data, 2003), Odonata (Donnelly, 2004a, 2004b, 2004c; C. Schiffer, State College, Pa., unpub. data, 2003), Tipulidae (Young and Gelhaus, 2000), Amphipoda and Isopoda (D. Glazier, Huntingdon, Pa., unpub. data, 2003) and crayfish (T. Nuttall, Lock Haven, Pa., unpub. data, 2003).

The phylogenetic schemes employed in the checklist mostly followed those of Smith (2001) and Thorp and Covich (2001) for the non-insect arthropods and Merritt and Cummins (1996) for the insects. Some groups such as the annelids (Kathman and Brinkhurst, 1998; Wetzel, 2001), water mites (Smith, 2001; Smith and Cook, 1991), mayflies (McCafferty, 2000), stoneflies (Stewart and Stark, 2002), caddisflies (Wiggins, 1995), beetles (Epler, 1996) and midges (Epler, 1995, 2001; R. Bode, New York Department of Environmental Conserva- tion, Albany, N.Y., written commun., 2001) required additional research to document the most current phylogeny. Although the senior author has extensively collected aquatic invertebrates within the Delaware River Basin, it was not possible to confirm all identifications of animals comprising the list. All taxa were reviewed for distributional accuracy (several taxa remain unresolved) and amended as necessary for synonomy and to correct "outdated taxonomy." Any weaknesses within certain groups in the checklist likely resulted from the perceived difficulty in conducting identifications, especially for the oligochaetes, mollusks, chironomids, and several minor phyla.

\section{Aquatic Invertebrates of the Delaware River Basin}

The compilation of a Delaware River Basin aquatic-invertebrate checklist incorporating multiple agency data (three county, four state, and four Federal) at 1,080 sites presented a challenge. Although these agencies have biomonitoring as a common goal, there was much latitude in the sampling designs, methods and seasons of collection, sample-site density, and levels of taxonomic resolution. Because this is a "working list" of taxa, the authors decided not to include data collected outside the 13 studies; however, the works of active taxonomists are mentioned in the methods section and their works will aid greatly in the assemblage of a comprehensive list of Delaware River Basin aquatic invertebrates.

At the end of 2000, the checklist consisted of 11 phyla comprising 20 classes, 46 orders, 196 families, 685 genera, and 835 species (table 2). An examination of the checklist revealed a number of interesting points. The arthropods made up about 83 percent of the total genera listed, and 95 percent of those were insects. Thirty percent of the total genera were dipterans; 62 percent of those were chironomids, which themselves made up 18 percent of the total genera. The mayflies, stoneflies, and caddisflies, prominent animals in biomonitoring assessments, collectively made up 24 percent of the total genera listed. Twenty-eight genera were listed as collected in at least 10 of the 13 studies and 10 genera were collected in 12 studies-Baetis, Isonychia, Stenonema, Sialis, Glossosoma, Polycentropus, Cheumatopsyche, Lepidostoma, Antocha, and Tipula.

Assessments of the status of the Delaware River Basin invertebrate assemblage are difficult to interpret at this time. As the diversity is better defined and other basins like the Susquehanna River are documented for comparison, a more complete understanding of how large rivers function in their benthic ecology will evolve. For now, it is hoped this updated taxa checklist will provide a consistent basis for current and future regional synthesis or comparison of biomonitoring data. 
Table 1. Agencies and programs from which benthic-invertebrate data were obtained for checklist of taxa in the Delaware River Basin.

[Number of sites sampled, and years during which sampling was conducted, pertain only to those data used in this report. DNREC, Delaware Department of Natural Resources and Environmental Control; STORET, U.S. Environmental Protection Agency Storage and Retrieval System; NJDEP, New Jersey Department of Environmental Protection; NYSDEC, New York State Department of Environmental Conservation; PaDEP, Pennsylvania Department of Environmental Protection; USGS, U.S. Geological Survey]

\begin{tabular}{|c|c|c|c|c|}
\hline \multirow{2}{*}{ Agency and program(s) } & \multirow{2}{*}{ Sites } & \multirow{2}{*}{ Year(s) } & \multicolumn{2}{|l|}{ Reference(s) } \\
\hline & & & Data source & Other references \\
\hline $\begin{array}{l}\text { Chester County Water Resources Authority } \\
\text { Stream Conditions of Chester County Program }\end{array}$ & 39 & $1990-2000$ & Reif, 1999; 2000; written commun., 2001 & - \\
\hline $\begin{array}{l}\text { Delaware Department of Natural Resources and Environ- } \\
\text { mental Control Macroinvertebrate Survey }\end{array}$ & 52 & 1993 & John Maxted, DNREC, written commun., 1998 & - \\
\hline $\begin{array}{l}\text { Delaware River Basin Commission } \\
\text { Scenic Rivers Monitoring Program }\end{array}$ & 19 & $1990-92$ & STORET & - \\
\hline $\begin{array}{l}\text { Monroe County Planning Commission } \\
\text { Water Quality Studies }\end{array}$ & 92 & $1990-2000$ & Monroe County Planning Commission, 1990-2000 & - \\
\hline $\begin{array}{l}\text { New Jersey Department of Environmental Protection } \\
\text { Ambient Biomonitoring Network }\end{array}$ & 328 & $1990-2000$ & $\begin{array}{l}\text { John Kurtz, NJDEP, written commun., 1995; 1996; } \\
2001\end{array}$ & $\begin{array}{l}\text { Kennen, 1999; Kurtenbach, } \\
\text { 1991; NJDEP, 1994a, 1994b, } \\
\text { 1996, 1999a, 1999b }\end{array}$ \\
\hline $\begin{array}{l}\text { New York State Department of Environmental Conservatio } \\
\text { Rotating Intensive Basins Study Program }\end{array}$ & 156 & $1993-2000$ & $\begin{array}{l}\text { Margaret Novak, NYSDEC, written commun., 1998, } \\
2001\end{array}$ & $\begin{array}{l}\text { Bode and others, 1993a, } \\
\text { 1993b, 1995a, 1995b, 1995c, } \\
\text { 2004; NYSDEC, } 1996\end{array}$ \\
\hline $\begin{array}{l}\text { Pennsylvania Department of Environmental Protection } \\
\text { Surface Water Quality Monitoring Network } \\
\text { Anti-Degradation Program }\end{array}$ & $\begin{array}{r}33 \\
150\end{array}$ & $\begin{array}{c}1991-96 \\
1990,1992-97\end{array}$ & $\begin{array}{l}\text { Rodney Kime, PaDEP, written commun., } 1998 \\
\text { Rodney Kime, PaDEP, written commun., } 1998\end{array}$ & Shertzer and Schreffler, 1996 \\
\hline $\begin{array}{l}\text { Pike County Conservation District } \\
\text { Surface Water Quality Monitoring Program }\end{array}$ & 44 & $1991-2000$ & Pike County Conservation District, 1991-2000 & - \\
\hline $\begin{array}{l}\text { U.S. Environmental Protection Agency } \\
\text { Environmental Monitoring and Assessment Program } \\
\text { Region } 3\end{array}$ & $\begin{array}{l}32 \\
25\end{array}$ & $\begin{array}{l}1993-96 \\
1994-96\end{array}$ & $\begin{array}{l}\text { U.S. Environmental Protection Agency, } 2000 \\
\text { Passmore and Green, } 1997\end{array}$ & Klemm and Lazorchak, 1994 \\
\hline $\begin{array}{l}\text { U.S. Geological Survey, Biological Resources Discipline } \\
\text { Hemlock/ Hardwood Study }\end{array}$ & 28 & 1997 & Snyder and others, 1999 & - \\
\hline $\begin{array}{l}\text { U.S. Geological Survey, Water Resources Discipline } \\
\text { National Water-Quality Assessment Program } \\
\text { Neversink Watersheds Studies } \\
\text { Other New York District Studies }\end{array}$ & $\begin{array}{l}35 \\
21 \\
26\end{array}$ & $\begin{array}{c}1999-2000 \\
1991-92,1995-99 \\
1997\end{array}$ & $\begin{array}{l}\text { unpublished data } \\
\text { Barry Baldigo, USGS, written commun., } 1999 \\
\text { Barry Baldigo, USGS, written commun., } 1999\end{array}$ & $\begin{array}{l}\text { Fischer, } 2004 \\
\text { Lawrence and others, } 1994\end{array}$ \\
\hline
\end{tabular}




\section{Acknowledgments}

This checklist could not have been completed without the help of the biologists from the selected agencies, Robert W. Bode (colleague reviewer) and Margaret A. Novak and Larry E. Abele from the New York State Department of Environmental Conservation, Albany, N.Y.; Rodney Kime from the Pennsylvania Department of Environmental Protection, Harrisburg, Pa.; Robert M. Ross from the U.S. Geological Survey, Biological Resources Discipline, Wellsboro, Pa.; Andrew G. Reif from the U.S. Geological Survey, Water Resources Discipline, Malvern, Pa.; Barry P. Baldigo from the U.S. Geological Survey, Water Resources Discipline, Troy, N.Y.; Allison R. Brigham, U.S. Geological Survey, Denver, Colo.; Eric Bartolacci from the Monroe County Planning Commission, Stroudsburg, Pa.; Sally Jones and Hannelore Schanzenbacher from the Pike County Conservation District, Hawley, Pa.; John R. Maxted from the Auckland Regional Council, Auckland, New Zealand, formerly of the Delaware Department of Natural Resources and Environmental Control, Dover, Del.; Edward D. Santoro and
Robert L. Limbeck from the Delaware River Basin Commission, Trenton, N.J.; Richard Evans from the National Park Service, Milford, Pa.; Al Korndoerfer and John Kurtz from the New Jersey Department of Environmental Protection, Trenton, N.J.; Ellyn Del Corso Campbell currently with Gannett Fleming, Camp Hill, Pa.; and Jim Green (retired) and Margaret Passmore from the U.S. Environmental Protection Agency, Wheeling, W.Va.

The authors especially thank Jonathan G. Kennen from the U.S. Geological Survey, Water Resources Discipline, Trenton, N.J., who served as a colleague reviewer and technical and taxonomic expert; Robin A. Brightbill, U.S. Geological Survey, Water Resources Discipline, New Cumberland, Pa., for data analysis, organization, and guidance; J. Kent Crawford, U.S. Geological Survey, New Cumberland, Pa., for editorial review; and Jeffrey M. Fischer, U.S. Geological Survey, Water Resources Discipline, Trenton, N.J., who served as Project Chief of the Delaware River Basin NAWQA Project for his support. 


\section{A Checklist of the Aquatic Invertebrates of the Delaware River Basin, 1990-2000}

Table 2. Checklist of the aquatic invertebrates of the Delaware River Basin, 1990-2000.

[Agency codes: C1, Chester County Water Resources Authority; C2, Monroe County Planning Commission; C3,Pike County Conservation District; F1,U.S. Environmental Protection Agency (USEPA), EMAP program; F2, USEPA Region 3; F3, Delaware River Basin Commission; F4, U.S. Geological Survey (USGS) Biological Resources Discipline; F5, USGS Water Resources Discipline (WRD) National Water-Quality Assessment Program; F6, USGS WRD, New York District; S1, Delaware Department of Natural Resources and Environmental Control; S2, New Jersey Department of Environmental Protection; S3, New York State Department of Environmental Conservation; S4, Pennsylvania Department of Environmental Protection]

\section{Taxonomic name}

Agency code

\begin{tabular}{|c|c|}
\hline PORIFERA & F5 \\
\hline \multicolumn{2}{|l|}{ DEMOSPONGIAE } \\
\hline \multicolumn{2}{|l|}{ HAPLOSCLERIDA } \\
\hline \multicolumn{2}{|l|}{ Spongillidae } \\
\hline Anheteromeyenia argyrosperma & S2 \\
\hline Ephydatia fluviatilis & S2 \\
\hline Eunapius fragilis & S2 \\
\hline Eunapius ingloviformis & S2 \\
\hline Heteromeyenia & S2 \\
\hline Heteromeyenia latitenta & S2 \\
\hline Heteromeyenia tubisperma & S2 \\
\hline Spongilla & S2 \\
\hline Spongilla lacustris & S2 \\
\hline Trochospongilla & S2 \\
\hline CNIDARIA & F6 \\
\hline \multicolumn{2}{|l|}{ HYDROZOA } \\
\hline \multicolumn{2}{|l|}{ HYDROIDA } \\
\hline \multicolumn{2}{|l|}{ Clavidae } \\
\hline Cordylophora lacustris & S2 \\
\hline \multicolumn{2}{|l|}{ Hydridae } \\
\hline Hydra & $\mathrm{C} 1, \mathrm{~F} 5, \mathrm{~S} 2$ \\
\hline PLATYHELMINTHES & $\mathrm{S} 2$ \\
\hline TURBELLARIA & $\mathrm{F} 1, \mathrm{~F} 2, \mathrm{~F} 5, \mathrm{~F} 6, \mathrm{~S} 2, \mathrm{~S} 4$ \\
\hline \multicolumn{2}{|l|}{ MACROSTOMIDA } \\
\hline \multicolumn{2}{|l|}{ Macrostomidae } \\
\hline Macrostomum & $\mathrm{C} 3$ \\
\hline \multicolumn{2}{|l|}{ PRORHYNCHIDA } \\
\hline \multicolumn{2}{|l|}{ Prorhynchidae } \\
\hline Geocentrophora baltica & $\mathrm{S} 2$ \\
\hline \multicolumn{2}{|l|}{ PROSERIATA } \\
\hline \multicolumn{2}{|l|}{ Plagiostomidae } \\
\hline Hydrolimax & S2 \\
\hline Hydrolimax grisea & S2 \\
\hline TRICLADIDA & S3 \\
\hline \multicolumn{2}{|l|}{ Dendrocoelidae } \\
\hline Procotyla & S2 \\
\hline
\end{tabular}

\section{Taxonomic name}

Agency code

\begin{tabular}{|c|c|}
\hline Procotyla fluviatilis & $\mathrm{S} 2$ \\
\hline \multicolumn{2}{|l|}{ Dugesiidae } \\
\hline Cura & $\mathrm{S} 4$ \\
\hline Cura foremanii & $\mathrm{S} 1, \mathrm{~S} 2$ \\
\hline Dugesia & $\mathrm{S} 2, \mathrm{~S} 4$ \\
\hline Dugesia tigrina & $\mathrm{S} 2$ \\
\hline Planariidae & $\mathrm{C} 1, \mathrm{~S} 4$ \\
\hline Hymanella retenuova & $\mathrm{S} 2$ \\
\hline Phagocata & $\mathrm{S} 2$ \\
\hline Phagocata gracilis & $\mathrm{S} 2$ \\
\hline Phagocata morgani morgani & $\mathrm{F} 1, \mathrm{~S} 2$ \\
\hline Phagocata velata & $\mathrm{S} 2$ \\
\hline Phagocata woodworthi & $\mathrm{S} 2$ \\
\hline Planaria & $\mathrm{C} 2, \mathrm{~S} 4$ \\
\hline NEMERTEA & $\mathrm{C} 1, \mathrm{~S} 2, \mathrm{~S} 4$ \\
\hline \multicolumn{2}{|l|}{ ENOPLA } \\
\hline \multicolumn{2}{|l|}{ HOPLONEMERTEA } \\
\hline \multicolumn{2}{|l|}{ Tetrastemmatidae } \\
\hline Prostoma & $\mathrm{C} 1, \mathrm{~F} 5, \mathrm{~S} 2$ \\
\hline Prostoma graecense & $\mathrm{S} 2, \mathrm{~S} 3$ \\
\hline NEMATODA & $\mathrm{C} 1, \mathrm{C} 2, \mathrm{~F} 1, \mathrm{~F} 5, \mathrm{~F} 6, \mathrm{~S} 2, \mathrm{~S} 4$ \\
\hline NEMATOMORPHA & $\mathrm{C} 2, \mathrm{~F} 3$ \\
\hline \multicolumn{2}{|l|}{ GORDIOIDEA } \\
\hline \multicolumn{2}{|l|}{ GORDIOIDA } \\
\hline \multicolumn{2}{|l|}{ Gordiidae } \\
\hline Gordius & $\mathrm{S} 4$ \\
\hline ECTOPROCTA & F5 \\
\hline \multicolumn{2}{|l|}{ GYMNOLAEMATA } \\
\hline \multicolumn{2}{|l|}{ CTENOSTOMATA } \\
\hline \multicolumn{2}{|l|}{ Paludicellidae } \\
\hline Paludicella & $\mathrm{S} 2$ \\
\hline Paludicella articulata & $\mathrm{S} 2$ \\
\hline \multicolumn{2}{|l|}{ PHYLACTOLAEMATA } \\
\hline \multicolumn{2}{|l|}{ PLUMATELLIDA } \\
\hline \multicolumn{2}{|l|}{ Fredericellidae } \\
\hline Fredericella & S2 \\
\hline Fredericella sultana & $\mathrm{S} 2$ \\
\hline \multicolumn{2}{|l|}{ Pectinatellidae } \\
\hline Pectinatella magnifica & $\mathrm{S} 2$ \\
\hline \multicolumn{2}{|l|}{ Plumatellidae } \\
\hline Hyalinella punctata & $\mathrm{S} 2$ \\
\hline Plumatella casmiana & $\mathrm{S} 2$ \\
\hline Plumatella fruticosa & $\mathrm{S} 2$ \\
\hline Plumatella repens & $\mathrm{S} 2$ \\
\hline ANNELIDA & $\mathrm{C} 2, \mathrm{~F} 3, \mathrm{~S} 4$ \\
\hline POLYCHAETA & $\mathrm{C} 1$ \\
\hline \multicolumn{2}{|l|}{ SABELLIDA } \\
\hline Sabellidae & $\mathrm{C} 1$ \\
\hline
\end{tabular}




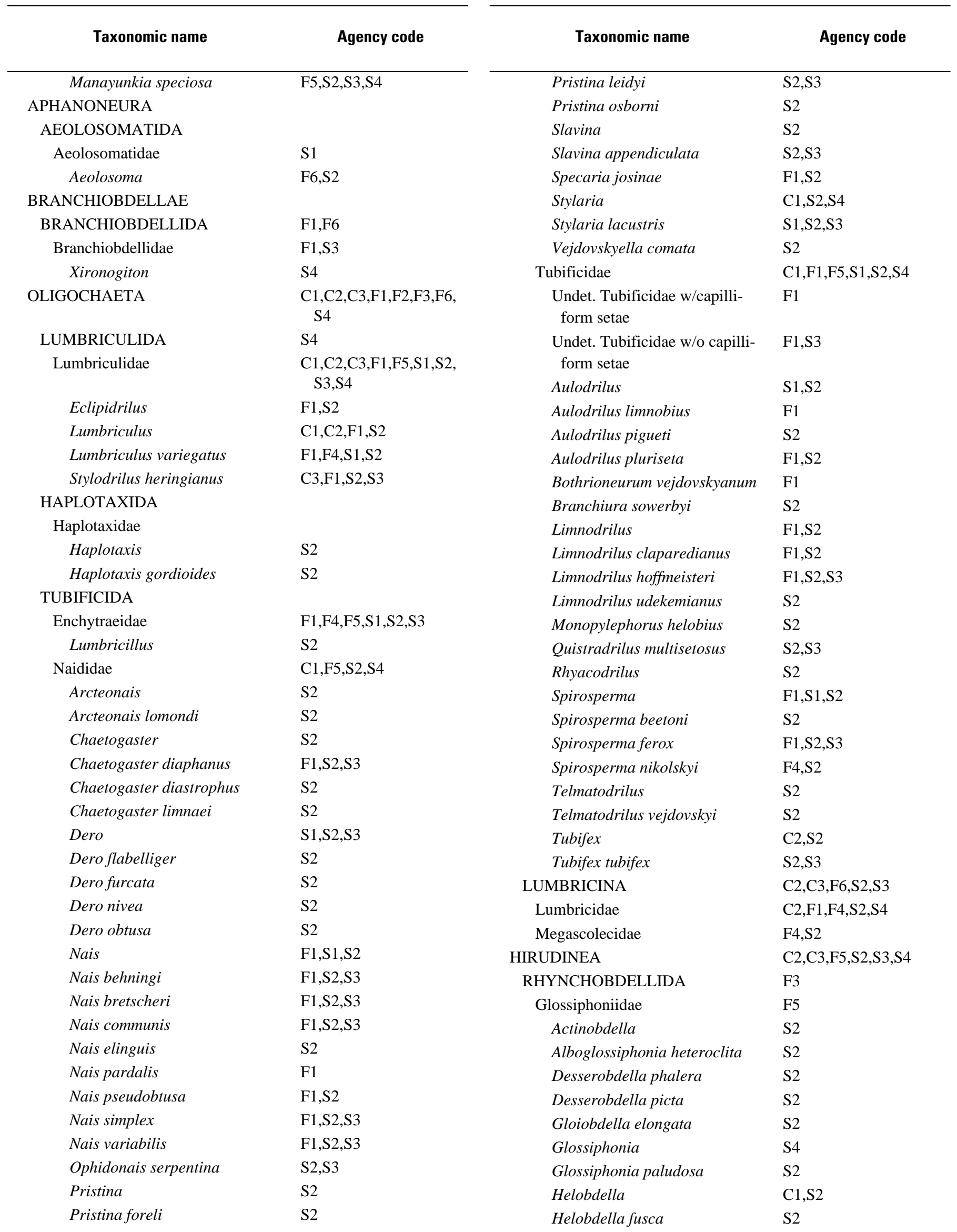




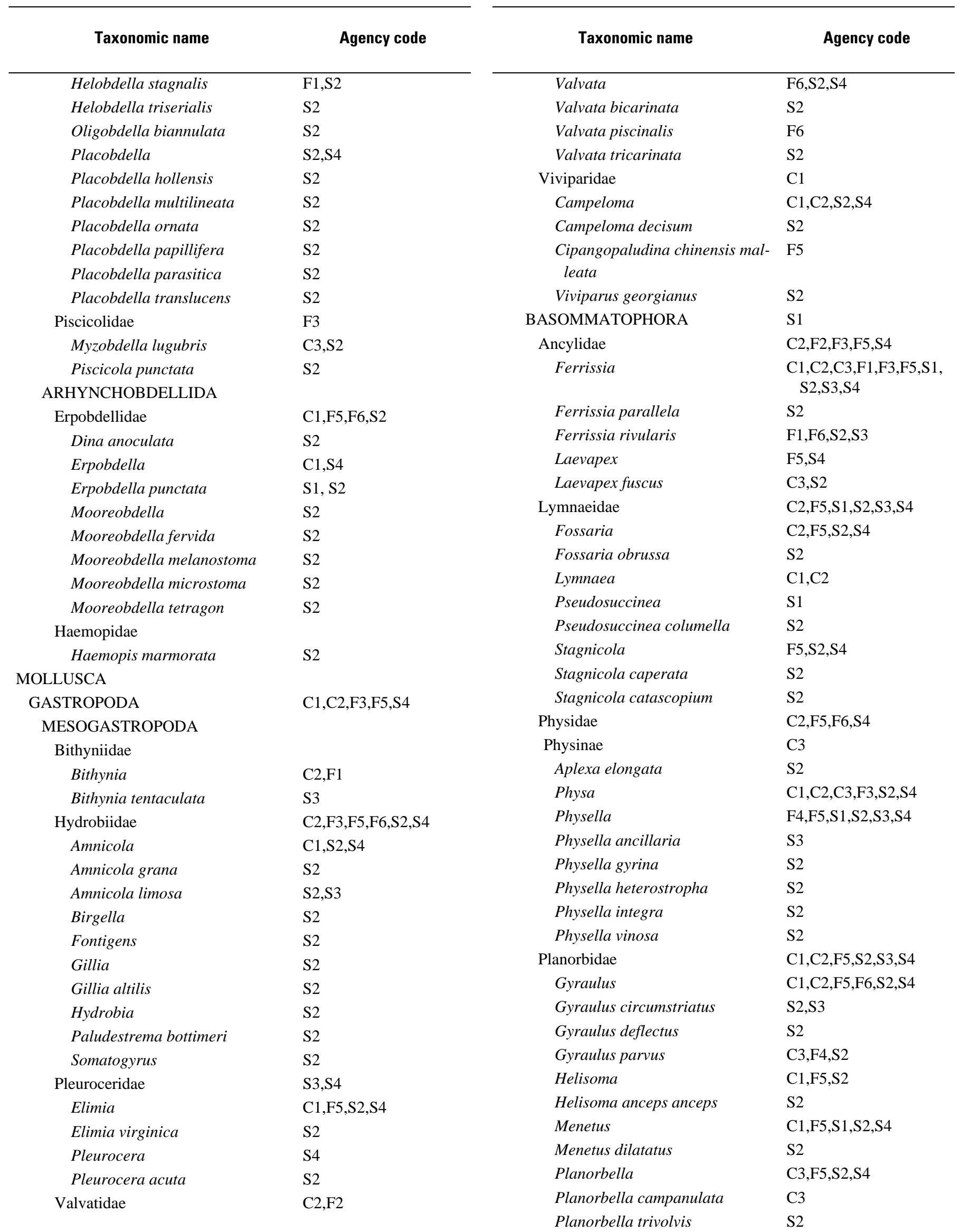




\begin{tabular}{|c|c|c|c|}
\hline Taxonomic name & Agency code & Taxonomic name & Agency code \\
\hline Planorbula & $\mathrm{C} 1, \mathrm{~S} 2, \mathrm{~S} 4$ & ARACHNIDA & \\
\hline Promenetus & $\mathrm{S} 2$ & ACARI & $\mathrm{F} 5, \mathrm{~F} 6$ \\
\hline Promenetus exacuous & S2 & HYDRACHNIDIA & $\mathrm{C} 1, \mathrm{~F} 1, \mathrm{~F} 6, \mathrm{~S} 4$ \\
\hline STYLOMMATOPHORA & & TROMBIDIFORMES & \\
\hline Discidae & & Eylaoidea & \\
\hline Discus cronkhitei & S2 & Limnocharidae & \\
\hline Helicodiscidae & & Limnochares & F1 \\
\hline Helicodiscus parallelus & S2 & Hydryphantoidea & \\
\hline Zonitidae & & Hydryphantidae & F4 \\
\hline Zonitoides & S2 & Hydrodromidae & \\
\hline Zonitoides arboreus & $\mathrm{S} 2$ & Hydrodroma despiciens & S2 \\
\hline BIVALVIA & $\mathrm{F} 1, \mathrm{~F} 3, \mathrm{~F} 5, \mathrm{~F} 6$ & Lebertioidea & \\
\hline UNIONOIDA & & Sperchonidae & \\
\hline Unionidae & $\mathrm{C} 2, \mathrm{~F} 5, \mathrm{~S} 2$ & Sperchon & $\mathrm{S} 2, \mathrm{~S} 4$ \\
\hline Alasmidonta undulata & S2 & Sperchonopsis verrucosa & S2 \\
\hline Anodonta & $\mathrm{S} 2$ & Lebertiidae & \\
\hline Anodonta imbecillis & F5 & Lebertia & $\mathrm{F} 1, \mathrm{~S} 1, \mathrm{~S} 2$ \\
\hline Anodonta implicata & $\mathrm{S} 2$ & Oxidae & \\
\hline Elliptio & F5 & Oxus & $\mathrm{C} 3$ \\
\hline Elliptio complanata & $\mathrm{F} 3, \mathrm{~S} 2$ & Torrenticolidae & \\
\hline Pyganodon cataracta & S2 & Torrenticola & $\mathrm{F} 4$ \\
\hline VENEROIDA & & Hygrobatoidea & \\
\hline Corbiculidae & & Limnesiidae & \\
\hline Corbicula & $\mathrm{F} 5, \mathrm{~S} 2, \mathrm{~S} 4$ & Limnesia & S2 \\
\hline Corbicula fluminea & $\mathrm{F} 3, \mathrm{~S} 1, \mathrm{~S} 2$ & Hygrobatidae & \\
\hline Sphaeriidae & $\begin{array}{l}\mathrm{C} 1, \mathrm{C} 2, \mathrm{C} 3, \mathrm{~F} 1, \mathrm{~F} 2, \mathrm{~F} 5, \mathrm{~F} 6, \\
\text { S2,S3,S4 }\end{array}$ & $\begin{array}{r}\text { Hygrobates } \\
\text { Unionicolidae }\end{array}$ & S2 \\
\hline Musculium & $\begin{array}{l}\mathrm{C} 1, \mathrm{C} 2, \mathrm{C} 3, \mathrm{~F} 1, \mathrm{~F} 3, \mathrm{~F} 5, \mathrm{~S} 1, \\
\text { S2,S4 }\end{array}$ & $\begin{array}{l}\text { Neumania } \\
\text { Unionicola }\end{array}$ & $\begin{array}{l}\mathrm{S} 2 \\
\mathrm{~S} 2\end{array}$ \\
\hline Musculium partumeium & $\mathrm{S} 2$ & Pionidae & \\
\hline Musculium securis & S2 & Piona & S2 \\
\hline Musculium transversum & $\mathrm{S} 2$ & Aturidae & \\
\hline Pisidium & $\begin{array}{l}\mathrm{C} 1, \mathrm{C} 2, \mathrm{C} 3, \mathrm{~F} 1, \mathrm{~F} 3, \mathrm{~F} 5, \mathrm{~S} 1, \\
\text { S2,S4 }\end{array}$ & $\begin{array}{l}\text { Brachypoda } \\
\text { Arrenuroidea }\end{array}$ & S2 \\
\hline Pisidium casertanum & $\mathrm{F} 4, \mathrm{~S} 2$ & $\begin{array}{l}\text { Arrenuroidea } \\
\text { Krendowskiidae }\end{array}$ & \\
\hline Pisidium compressum & S2 & Geayia ovata & S2 \\
\hline Pisidium dubium & $\mathrm{F} 1, \mathrm{~S} 2$ & $\begin{array}{l}\text { Geayla ovata } \\
\text { Arrenuridae }\end{array}$ & \\
\hline Pisidium fallax & $\mathrm{S} 2$ & Arrenurus & S2 \\
\hline Pisidium punctatum & $\mathrm{S} 2$ & ORIBATIDA & \\
\hline Pisidium variabile & S2 & Hydrozetidae & \\
\hline Pisidium walkeri & $\mathrm{S} 2$ & Hydrozetes & $\mathrm{S} 1, \mathrm{~S} 2$ \\
\hline Sphaerium & $\begin{array}{l}\mathrm{C} 1, \mathrm{C} 2, \mathrm{C} 3, \mathrm{~F} 3, \mathrm{~F} 5, \mathrm{~S} 2, \mathrm{~S} 3, \\
\text { S4 }\end{array}$ & ARTHROPODA & \\
\hline Sphaerium fabale & $\mathrm{S} 2$ & CRUSTACEA & $\mathrm{C} 2, \mathrm{~F} 6$ \\
\hline Sphaerium occidentale & $\mathrm{S} 2$ & BRANCHIOPODA & \\
\hline Sphaerium rhomboideum & $\mathrm{S} 2$ & CLADOCERA & $\mathrm{C} 1$ \\
\hline Sphaerium simile & S2 & Chydoridae & \\
\hline Sphaerium striatinum & $\mathrm{S} 2$ & Eurycercus lamellatus & S2 \\
\hline
\end{tabular}




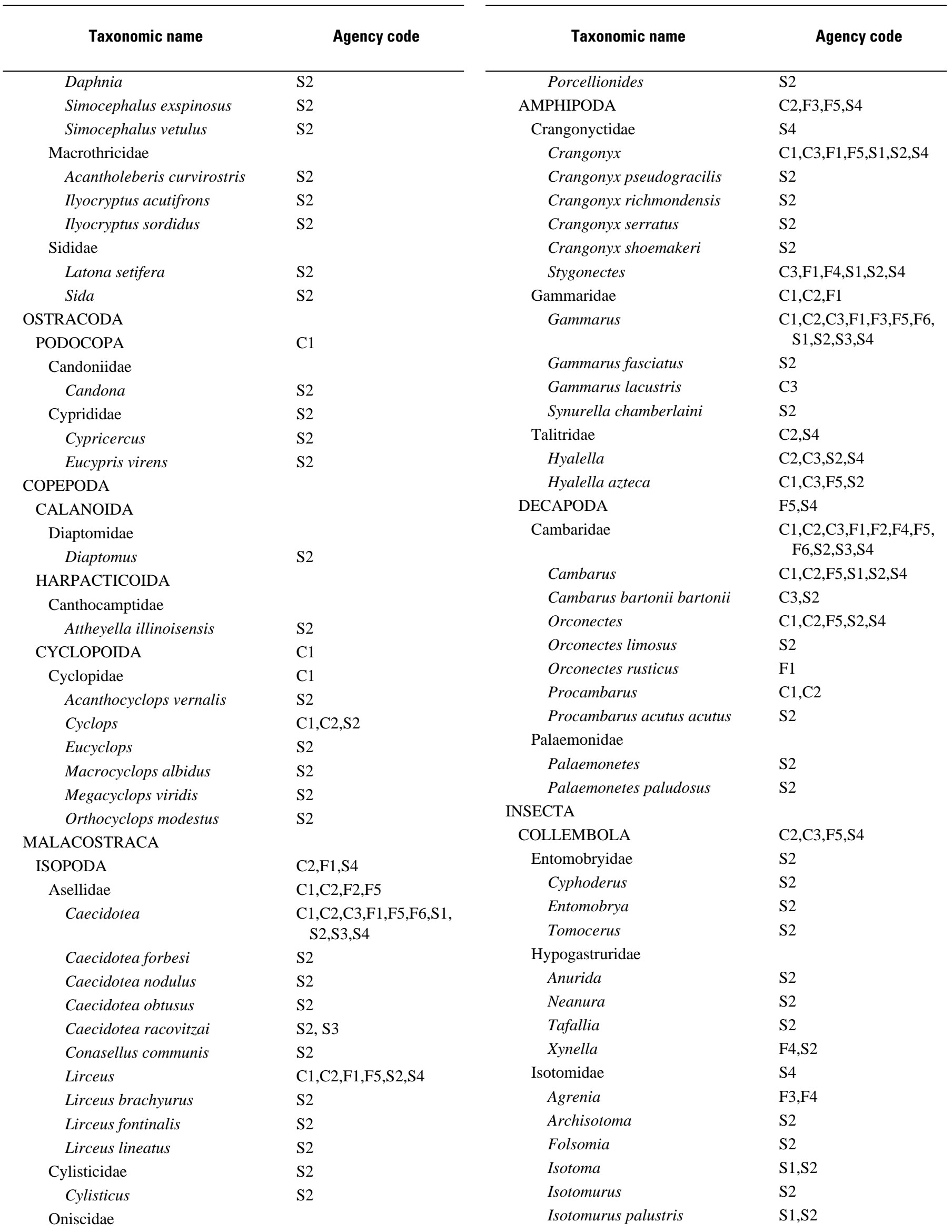


Taxonomic name

\begin{tabular}{|c|c|}
\hline \multicolumn{2}{|l|}{ Onychiuridae } \\
\hline Onychiurus & $\mathrm{S} 2$ \\
\hline \multicolumn{2}{|l|}{ Poduridae } \\
\hline Podura & $\mathrm{S} 4$ \\
\hline Podura aquatica & $\mathrm{S} 2$ \\
\hline EPHEMEROPTERA & $\mathrm{C} 2, \mathrm{~F} 1, \mathrm{~F} 3, \mathrm{~F} 5, \mathrm{~F} 6$ \\
\hline \multicolumn{2}{|l|}{ CARAPACEA } \\
\hline \multicolumn{2}{|l|}{ Prosopistomatoidea } \\
\hline \multicolumn{2}{|l|}{ Baetiscidae } \\
\hline Baetisca & $\mathrm{C} 2, \mathrm{~F} 3, \mathrm{~F} 6, \mathrm{~S} 2, \mathrm{~S} 3$ \\
\hline Baetisca carolina & $\mathrm{S} 2$ \\
\hline Baetisca obesa & $\mathrm{S} 2$ \\
\hline \multicolumn{2}{|l|}{ FURCATERGALIA } \\
\hline \multicolumn{2}{|l|}{ Leptophlebioidea } \\
\hline Leptophlebiidae & $\begin{array}{l}\mathrm{C} 1, \mathrm{C} 2, \mathrm{~F} 1, \mathrm{~F} 2, \mathrm{~F} 5, \mathrm{~F} 6, \mathrm{~S} 1, \\
\text { S2,S3,S4 }\end{array}$ \\
\hline Choroterpes & $\mathrm{S} 2$ \\
\hline Habrophlebia & $\mathrm{C} 1, \mathrm{~S} 4$ \\
\hline Habrophlebia vibrans & $\mathrm{F} 1, \mathrm{~S} 2$ \\
\hline Habrophlebiodes & $\mathrm{C} 3, \mathrm{~F} 1, \mathrm{~S} 4$ \\
\hline Leptophlebia & $\mathrm{S} 1, \mathrm{~S} 2, \mathrm{~S} 4$ \\
\hline Paraleptophlebia & $\begin{array}{c}\mathrm{C} 1, \mathrm{C} 2, \mathrm{C} 3, \mathrm{~F} 1, \mathrm{~F} 3, \mathrm{~F} 4, \mathrm{~F} 5, \\
\mathrm{~F} 6, \mathrm{~S} 2, \mathrm{~S} 3, \mathrm{~S} 4\end{array}$ \\
\hline Paraleptophlebia guttata & $\mathrm{F} 6, \mathrm{~S} 3$ \\
\hline Paraleptophlebia mollis & $\mathrm{C} 3, \mathrm{~F} 6, \mathrm{~S} 3$ \\
\hline Paraleptophlebia volitans & $\mathrm{F} 6, \mathrm{~S} 3$ \\
\hline \multicolumn{2}{|l|}{ Ephemeroidea } \\
\hline \multicolumn{2}{|l|}{ Potamanthidae } \\
\hline Anthopotamus & $\mathrm{C} 1, \mathrm{~F} 3, \mathrm{~S} 2, \mathrm{~S} 4$ \\
\hline Anthopotamus myops & $\mathrm{S} 1$ \\
\hline \multicolumn{2}{|l|}{ Polymitarcyidae } \\
\hline Ephoron leukon & $\mathrm{F} 3, \mathrm{~S} 2, \mathrm{~S} 4$ \\
\hline Ephemeridae & $\mathrm{C} 2, \mathrm{~F} 1, \mathrm{~F} 2$ \\
\hline Ephemera & $\mathrm{C} 1, \mathrm{C} 3, \mathrm{~F} 1, \mathrm{~F} 3, \mathrm{~S} 2, \mathrm{~S} 3, \mathrm{~S} 4$ \\
\hline Ephemera guttulata & $\mathrm{C} 3$ \\
\hline Ephemera varia & $\mathrm{F} 1$ \\
\hline Hexagenia & $\mathrm{C} 2, \mathrm{~F} 3, \mathrm{~S} 2, \mathrm{~S} 4$ \\
\hline Litobrancha recurvata & $\mathrm{C} 3, \mathrm{~F} 1$ \\
\hline Pentagenia & F3 \\
\hline \multicolumn{2}{|l|}{ Caenoidea } \\
\hline Caenidae & $\mathrm{C} 2, \mathrm{~F} 1, \mathrm{~F} 2$ \\
\hline Brachycercus & F1,F5,F6 \\
\hline Caenis & $\begin{array}{l}\mathrm{C} 1, \mathrm{C} 2, \mathrm{~F} 1, \mathrm{~F} 3, \mathrm{~F} 5, \mathrm{~F} 6, \mathrm{~S} 1, \\
\text { S2,S3,S4 }\end{array}$ \\
\hline Caenis anceps & F5 \\
\hline Caenis cf. hilaris & F5 \\
\hline Caenis latipennis & S3 \\
\hline Caenis macafferti & S3 \\
\hline
\end{tabular}

Ephemerelloidea

Agency code
Taxonomic name

Agency code

\begin{tabular}{|c|c|}
\hline Ephemerellidae & $\begin{array}{l}\text { C2,C3,F1,F2,F3,F5,S1, } \\
\text { S3,S4 }\end{array}$ \\
\hline Attenella & $\mathrm{C} 3, \mathrm{~F} 3, \mathrm{~F} 6, \mathrm{~S} 4$ \\
\hline Attenella attenuata & $\mathrm{C} 3, \mathrm{~F} 5, \mathrm{~F} 6, \mathrm{~S} 2, \mathrm{~S} 3$ \\
\hline Attenella margarita & F6 \\
\hline Dannella & $\mathrm{F} 1, \mathrm{~F} 3, \mathrm{~F} 6, \mathrm{~S} 4$ \\
\hline Dannella lita & $\mathrm{F} 1, \mathrm{~S} 2$ \\
\hline Dannella simplex & $\mathrm{C} 3, \mathrm{~F} 1, \mathrm{~S} 2$ \\
\hline Drunella & $\mathrm{C} 3, \mathrm{~F} 1, \mathrm{~F} 3, \mathrm{~F} 6, \mathrm{~S} 2, \mathrm{~S} 4$ \\
\hline Drunella allegheniensis & $\mathrm{C} 3$ \\
\hline Drunella cornuta & $\mathrm{F} 1, \mathrm{~F} 6, \mathrm{~S} 3$ \\
\hline Drunella cornuta/cornutella & $\mathrm{C} 3$ \\
\hline Drunella cornutella & $\mathrm{F} 1, \mathrm{~F} 6, \mathrm{~S} 2, \mathrm{~S} 3$ \\
\hline Drunella lata & $\mathrm{C} 2, \mathrm{C} 3, \mathrm{~F} 6, \mathrm{~S} 2, \mathrm{~S} 3$ \\
\hline Drunella tuberculata & $\mathrm{C} 3, \mathrm{~F} 1, \mathrm{~F} 5, \mathrm{~F} 6, \mathrm{~S} 3$ \\
\hline Drunella walkeri & $\mathrm{C} 3, \mathrm{~S} 2, \mathrm{~S} 3$ \\
\hline Ephemerella & $\begin{array}{l}\mathrm{C} 1, \mathrm{C} 2, \mathrm{C} 3, \mathrm{~F} 1, \mathrm{~F} 3, \mathrm{~F} 5, \mathrm{~F} 6, \\
\mathrm{~S} 1, \mathrm{~S} 2, \mathrm{~S} 3, \mathrm{~S} 4\end{array}$ \\
\hline Ephemerella aurivillii & S2 \\
\hline Ephemerella dorothea & $\mathrm{C} 3, \mathrm{~F} 1, \mathrm{~F} 4, \mathrm{~F} 6, \mathrm{~S} 2, \mathrm{~S} 3$ \\
\hline Ephemerella excrucians & $\mathrm{C} 3$ \\
\hline Ephemerella inconstans & $\mathrm{F} 1$ \\
\hline Ephemerella invaria & $\mathrm{C} 3, \mathrm{~F} 1, \mathrm{~S} 2, \mathrm{~S} 3$ \\
\hline Ephemerella invaria/rotunda & $\mathrm{C} 3, \mathrm{~F} 1$ \\
\hline Ephemerella needhami & $\mathrm{C} 3, \mathrm{~S} 2$ \\
\hline Ephemerella rotunda & $\mathrm{C} 3, \mathrm{~F} 1, \mathrm{~F} 4, \mathrm{~F} 6, \mathrm{~S} 2$ \\
\hline Ephemerella septentrionalis & $\mathrm{C} 3, \mathrm{~F} 1, \mathrm{~S} 2$ \\
\hline Ephemerella subvaria & $\mathrm{C} 3, \mathrm{~S} 2, \mathrm{~S} 3$ \\
\hline Ephemerella/Serratella & F6 \\
\hline Eurylophella & $\begin{array}{l}\mathrm{C} 1, \mathrm{C} 3, \mathrm{~F} 1, \mathrm{~F} 5, \mathrm{~F} 6, \mathrm{~S} 1, \mathrm{~S} 2, \\
\text { S3,S4 }\end{array}$ \\
\hline Eurylophella bicolor & $\mathrm{C} 3, \mathrm{~F} 1, \mathrm{~S} 2$ \\
\hline Eurylophella doris & $\mathrm{C} 3$ \\
\hline Eurylophella funeralis & $\mathrm{C} 3, \mathrm{~F} 1, \mathrm{~F} 4$ \\
\hline Eurylophella poconoensis & $\mathrm{C} 3$ \\
\hline Eurylophella temporalis & $\mathrm{C} 3, \mathrm{~F} 1, \mathrm{~S} 2$ \\
\hline Eurylophella temporalis/doris & $\mathrm{C} 3$ \\
\hline Eurylophella verisimilis & $\mathrm{C} 3, \mathrm{~F} 1$ \\
\hline Serratella & $\begin{array}{l}\mathrm{C} 1, \mathrm{C} 3, \mathrm{~F} 1, \mathrm{~F} 3, \mathrm{~F} 5, \mathrm{~F} 6, \mathrm{~S} 1, \\
\text { S2,S3,S4 }\end{array}$ \\
\hline Serratella deficiens & $\mathrm{C} 3, \mathrm{~F} 1, \mathrm{~F} 5, \mathrm{~F} 6, \mathrm{~S} 2, \mathrm{~S} 3$ \\
\hline Serratella frisoni & F5 \\
\hline Serratella serrata & $\mathrm{F} 1, \mathrm{~F} 5, \mathrm{~F} 6, \mathrm{~S} 2, \mathrm{~S} 3$ \\
\hline Serratella serratoides & $\mathrm{F} 6, \mathrm{~S} 2, \mathrm{~S} 3$ \\
\hline Leptohyphidae & $\mathrm{C} 1, \mathrm{C} 2, \mathrm{~F} 1, \mathrm{~F} 2, \mathrm{~F} 5$ \\
\hline Tricorythodes & $\begin{array}{l}\mathrm{C} 1, \mathrm{C} 2, \mathrm{C} 3, \mathrm{~F} 1, \mathrm{~F} 3, \mathrm{~F} 5, \mathrm{~F} 6, \\
\mathrm{~S} 1, \mathrm{~S} 2, \mathrm{~S} 3, \mathrm{~S} 4\end{array}$ \\
\hline
\end{tabular}

\section{PISCIFORMA}

Baetoidea 


\begin{tabular}{|c|c|c|c|}
\hline Taxonomic name & Agency code & Taxonomic name & Agency code \\
\hline $\begin{array}{r}\text { Ameletidae } \\
\text { Ameletus }\end{array}$ & $\begin{array}{l}\mathrm{C} 1, \mathrm{C} 2, \mathrm{C} 3, \mathrm{~F} 1, \mathrm{~F} 4, \mathrm{~F} 6, \mathrm{~S} 2, \\
\quad \mathrm{~S} 3, \mathrm{~S} 4\end{array}$ & Cinygmula & $\begin{array}{l}\mathrm{C} 2, \mathrm{~F} 1, \mathrm{~F} 2, \mathrm{~F} 3, \mathrm{~F} 5, \mathrm{~F} 6, \mathrm{~S} 1, \\
\text { S2,S3,S4 } \\
\text { C3,F1,S2,S4 }\end{array}$ \\
\hline Acentrella & $\begin{array}{l}\text { C2,F1,F2,F3,F5,S1,S3, } \\
\text { S4 } \\
\text { C3,F1,F3,F5,F6,S2,S3, } \\
\quad \text { S4 }\end{array}$ & $\begin{array}{l}\text { Cinygmula subaequalis } \\
\text { Epeorus } \\
\text { Epeorus (Iron) }\end{array}$ & $\begin{array}{l}\text { C3 } \\
\text { C1,C2,C3,F1,F3,F5,F6, } \\
\quad \text { S2,S4 } \\
\text { F4,S3 }\end{array}$ \\
\hline Acentrella ampla & $\mathrm{C} 3, \mathrm{~F} 1, \mathrm{~F} 6, \mathrm{~S} 1$ & Epeorus pleuralis/punctatus & $\mathrm{C} 3$ \\
\hline Acentrella turbida & $\mathrm{C} 3, \mathrm{~F} 5, \mathrm{~F} 6, \mathrm{~S} 2$ & Epeorus vitreus & $\mathrm{C} 3$ \\
\hline $\begin{array}{l}\text { Acerpenna } \\
\text { Acerpenna macdunnoughi }\end{array}$ & $\begin{array}{l}\text { C3,F5,S1,S4 } \\
\text { S3 }\end{array}$ & Heptagenia & $\begin{array}{l}\mathrm{C} 1, \mathrm{C} 2, \mathrm{C} 3, \mathrm{~F} 3, \mathrm{~F} 5, \mathrm{~F} 6, \mathrm{~S} 2, \\
\quad \mathrm{~S} 3, \mathrm{~S} 4\end{array}$ \\
\hline Baetis brunneicolor & $\begin{array}{l}\mathrm{C} 1, \mathrm{C} 2, \mathrm{C} 3, \mathrm{~F} 1, \mathrm{~F} 3, \mathrm{~F} 4, \mathrm{~F} 5, \\
\mathrm{~F} 6, \mathrm{~S} 1, \mathrm{~S} 2, \mathrm{~S} 3, \mathrm{~S} 4 \\
\mathrm{C} 3, \mathrm{~F} 6, \mathrm{~S} 2, \mathrm{~S} 3\end{array}$ & $\begin{array}{l}\text { Heptagenia pulla } \\
\text { Leucrocuta }\end{array}$ & $\begin{array}{l}\text { S3 } \\
\text { C3,F1,F3,F5,F6,S2,S3, } \\
\quad \text { S4 }\end{array}$ \\
\hline Baetis flavistriga & $\mathrm{C} 3, \mathrm{~F} 1, \mathrm{~F} 5, \mathrm{~F} 6, \mathrm{~S} 2, \mathrm{~S} 3$ & Leucrocuta hebe & $\mathrm{C} 3$ \\
\hline Baetis intercalaris & $\mathrm{C} 3, \mathrm{~F} 1, \mathrm{~F} 5, \mathrm{~F} 6, \mathrm{~S} 2, \mathrm{~S} 3$ & Macdunnoa & $\mathrm{C} 3$ \\
\hline Baetis pluto & $\mathrm{C} 3, \mathrm{~S} 3$ & Nixe & $\mathrm{C} 3, \mathrm{~F} 1, \mathrm{~F} 6, \mathrm{~S} 4$ \\
\hline Baetis tricaudatus & $\mathrm{C} 3, \mathrm{~F} 1, \mathrm{~F} 4, \mathrm{~F} 5, \mathrm{~F} 6, \mathrm{~S} 2, \mathrm{~S} 3$ & Nixe lucidipennis & $\mathrm{S} 2$ \\
\hline Callibaetis & $\mathrm{C} 2, \mathrm{~F} 1, \mathrm{~F} 3, \mathrm{~F} 5, \mathrm{~S} 2$ & Rhithrogena & $\mathrm{C} 3, \mathrm{~F} 4, \mathrm{~F} 6, \mathrm{~S} 4$ \\
\hline $\begin{array}{l}\text { Centroptilum } \\
\text { Cloeon }\end{array}$ & $\begin{array}{l}\mathrm{C} 1, \mathrm{C} 3, \mathrm{~F} 1, \mathrm{~F} 3, \mathrm{~F} 5, \mathrm{~S} 2, \mathrm{~S} 4 \\
\mathrm{C} 2, \mathrm{C} 3, \mathrm{~F} 1, \mathrm{~F} 3, \mathrm{~S} 2\end{array}$ & Stenacron & $\begin{array}{l}\mathrm{C} 1, \mathrm{C} 3, \mathrm{~F} 1, \mathrm{~F} 3, \mathrm{~F} 5, \mathrm{~S} 1, \mathrm{~S} 2, \\
\quad \mathrm{~S} 4\end{array}$ \\
\hline Diphetor hageni & $\mathrm{C} 3, \mathrm{~F} 6, \mathrm{~S} 2$ & Stenacron candidum & $\mathrm{S} 2$ \\
\hline Heterocloeon & $\mathrm{C} 3, \mathrm{~F} 3, \mathrm{~F} 5, \mathrm{~F} 6, \mathrm{~S} 2, \mathrm{~S} 4$ & Stenacron carolina & $\mathrm{C} 3$ \\
\hline Heterocloeon curiosum & F5 & Stenacron gildersleevi & $\mathrm{C} 3$ \\
\hline Paracloeodes & $\mathrm{S} 2$ & Stenacron interpunctatum & $\mathrm{C} 3, \mathrm{~F} 5, \mathrm{~F} 6, \mathrm{~S} 2, \mathrm{~S} 3$ \\
\hline Plauditus & $\mathrm{F} 5, \mathrm{~S} 2$ & Stenacron minnetonka & $\mathrm{C} 3, \mathrm{~S} 2$ \\
\hline Plauditus cestus & F6 & Stenacron pallidum & $\mathrm{C} 3, \mathrm{~S} 2$ \\
\hline Plauditus cingulatus & $\begin{array}{l}\mathrm{C} 3, \mathrm{~S} 2 \\
\mathrm{~S} 2\end{array}$ & Stenonema & $\begin{array}{l}\mathrm{C} 1, \mathrm{C} 2, \mathrm{C} 3, \mathrm{~F} 1, \mathrm{~F} 3, \mathrm{~F} 5, \mathrm{~F} 6, \\
\text { S1,S2,S3,S4 }\end{array}$ \\
\hline Plauditus parvulus & $\begin{array}{l}\mathrm{S} 2 \\
\mathrm{~S} 2\end{array}$ & Stenonema femoratum & $\mathrm{S} 3$ \\
\hline Plauditus punctiventris & $\mathrm{C} 3, \mathrm{~S} 2$ & Stenonema ithaca & $\mathrm{C} 3, \mathrm{~F} 1, \mathrm{~F} 5, \mathrm{~F} 6, \mathrm{~S} 2, \mathrm{~S} 3$ \\
\hline Procloeon & $\mathrm{F} 5, \mathrm{~F} 6, \mathrm{~S} 2$ & Stenonema luteum & $\mathrm{C} 3, \mathrm{~S} 2$ \\
\hline Pseudocloeon & $\mathrm{C} 1, \mathrm{C} 2, \mathrm{~F} 3, \mathrm{~F} 5, \mathrm{~S} 2, \mathrm{~S} 4$ & Stenonema mediopunctatum & $\mathrm{F} 5, \mathrm{~S} 2$ \\
\hline Pseudocloeon propinquum & $\mathrm{F} 5, \mathrm{~S} 2, \mathrm{~S} 3$ & mediopunctatum & \\
\hline Metretopodidae & & Stenonema meririvulanum & $\mathrm{F} 4$ \\
\hline Siphloplecton & $\mathrm{S} 2$ & Stenonema mexicanum integrum & $\mathrm{S} 2, \mathrm{~S} 3$ \\
\hline Siphlonuridae & $\mathrm{C} 2, \mathrm{~F} 1, \mathrm{~F} 2, \mathrm{~S} 1$ & Stenonema modestum & $\mathrm{C} 3, \mathrm{~F} 1, \mathrm{~F} 5, \mathrm{~F} 6, \mathrm{~S} 2, \mathrm{~S} 3$ \\
\hline Siphlonisca & $\mathrm{S} 4$ & Stenonema pudicum & $\mathrm{C} 3, \mathrm{~F} 1, \mathrm{~F} 4, \mathrm{~F} 6, \mathrm{~S} 2$ \\
\hline Siphlonurus & $\mathrm{C} 1, \mathrm{C} 3, \mathrm{~F} 3, \mathrm{~S} 2, \mathrm{~S} 3, \mathrm{~S} 4$ & Stenonema pulchellum & F5 \\
\hline SETISURA & & Stenonema terminatum & $\mathrm{F} 1, \mathrm{~F} 6, \mathrm{~S} 2, \mathrm{~S} 3$ \\
\hline Heptagenioidea & & Stenonema vicarium & $\mathrm{F} 4, \mathrm{~F} 5, \mathrm{~S} 2, \mathrm{~S} 3$ \\
\hline Isonychiidae & $\mathrm{C} 1, \mathrm{C} 2, \mathrm{~F} 1, \mathrm{~F} 2$ & ODONATA & $\mathrm{C} 2$ \\
\hline Isonychia & $\begin{array}{l}\mathrm{C} 1, \mathrm{C} 2, \mathrm{C} 3, \mathrm{~F} 1, \mathrm{~F} 3, \mathrm{~F} 4, \mathrm{~F} 5, \\
\mathrm{~F} 6, \mathrm{~S} 1, \mathrm{~S} 2, \mathrm{~S} 3, \mathrm{~S} 4\end{array}$ & $\begin{array}{l}\text { ANISOPTERA } \\
\text { Aeschnidae }\end{array}$ & $\begin{array}{l}\mathrm{F} 1 \\
\mathrm{C} 2, \mathrm{~F} 1, \mathrm{~F} 2, \mathrm{~F} 5\end{array}$ \\
\hline Isonychia arida & $\mathrm{S} 2$ & Aeschna & $\mathrm{C} 1, \mathrm{C} 2, \mathrm{~F} 5, \mathrm{~S} 2$ \\
\hline Isonychia bicolor & $\mathrm{S} 2, \mathrm{~S} 3$ & Aeschna interrupta & $\mathrm{S} 2$ \\
\hline Isonychia sayi & $\mathrm{S} 2$ & Anax & S2 \\
\hline & & Basiaeschna & $\mathrm{S} 2$ \\
\hline
\end{tabular}




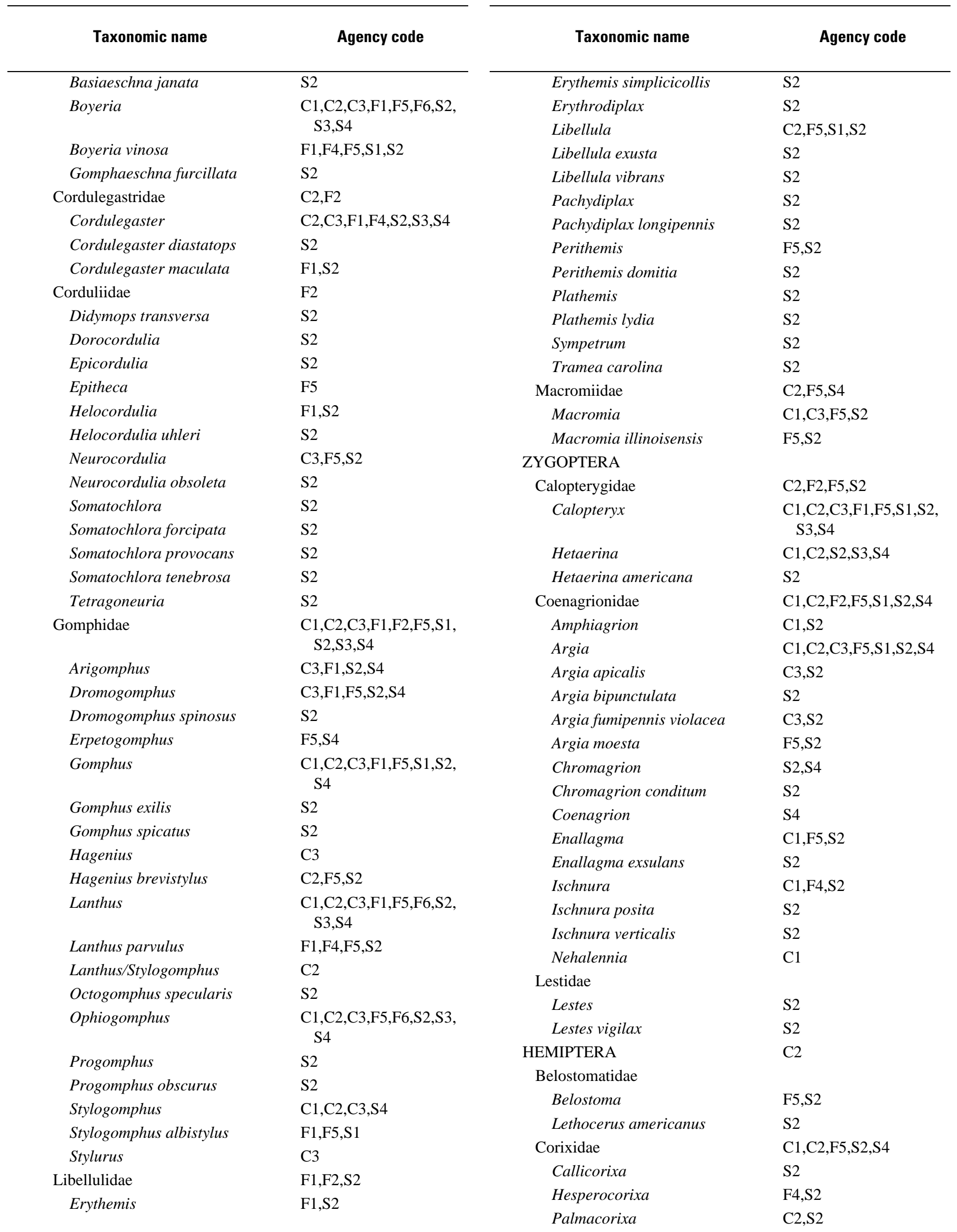




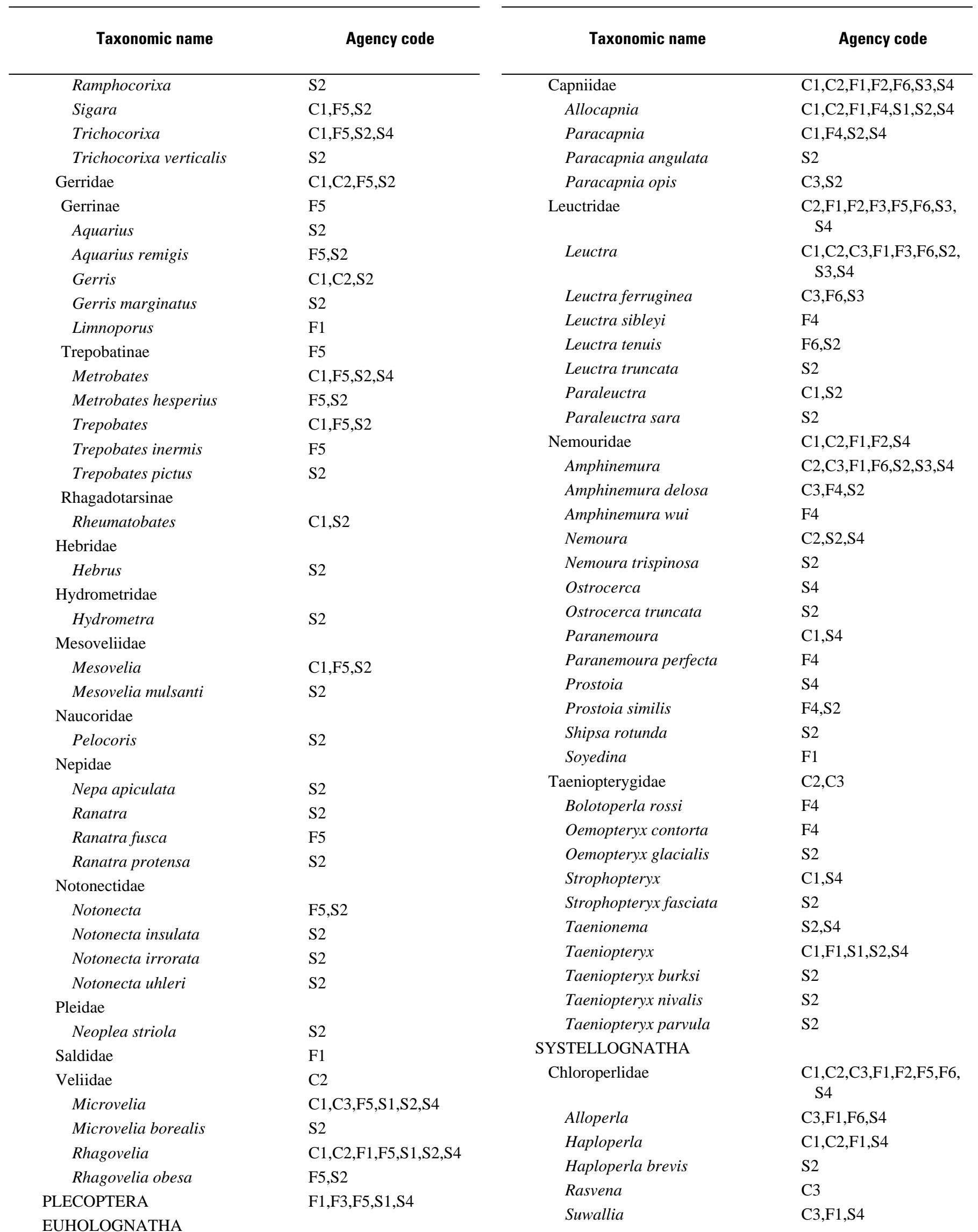




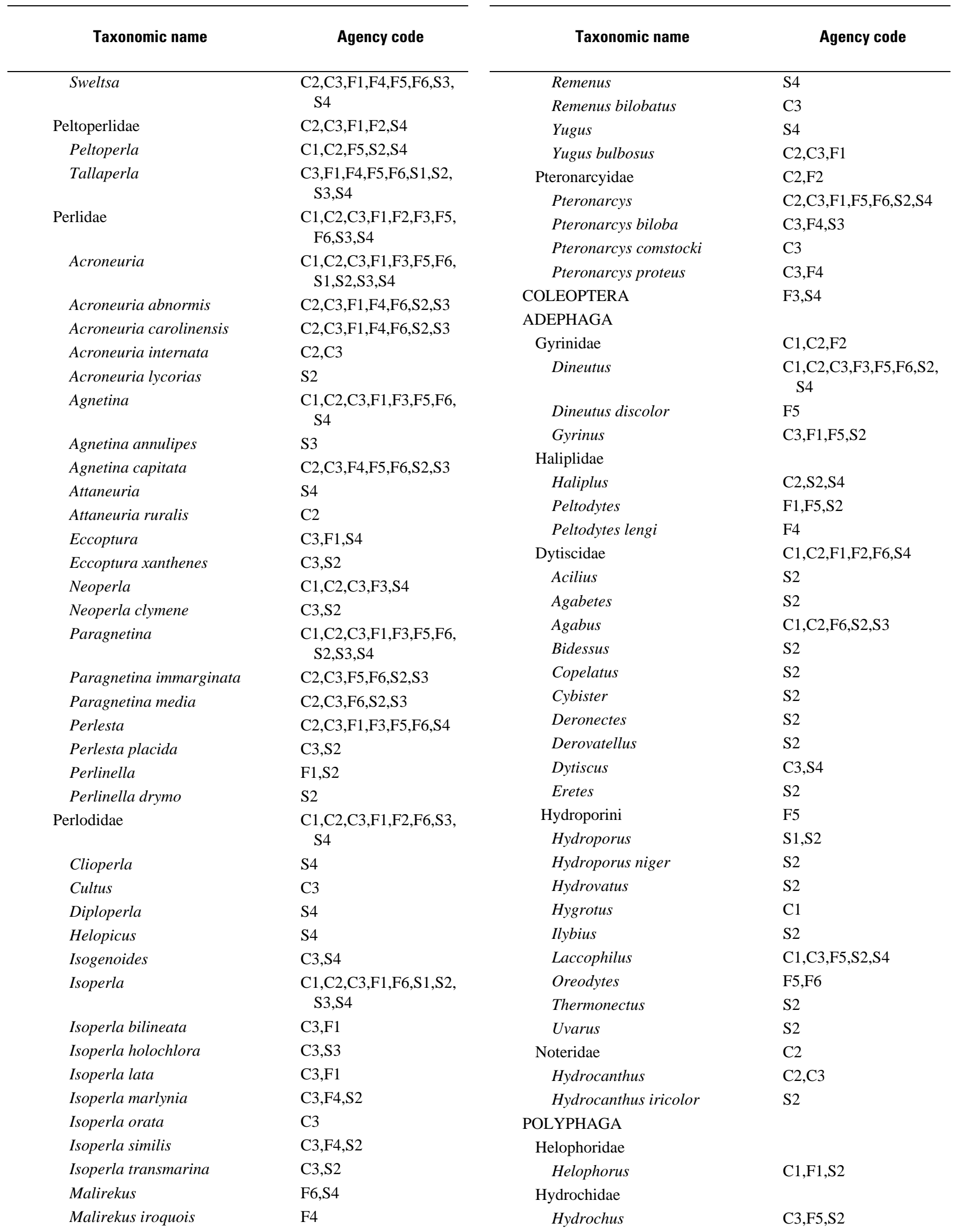




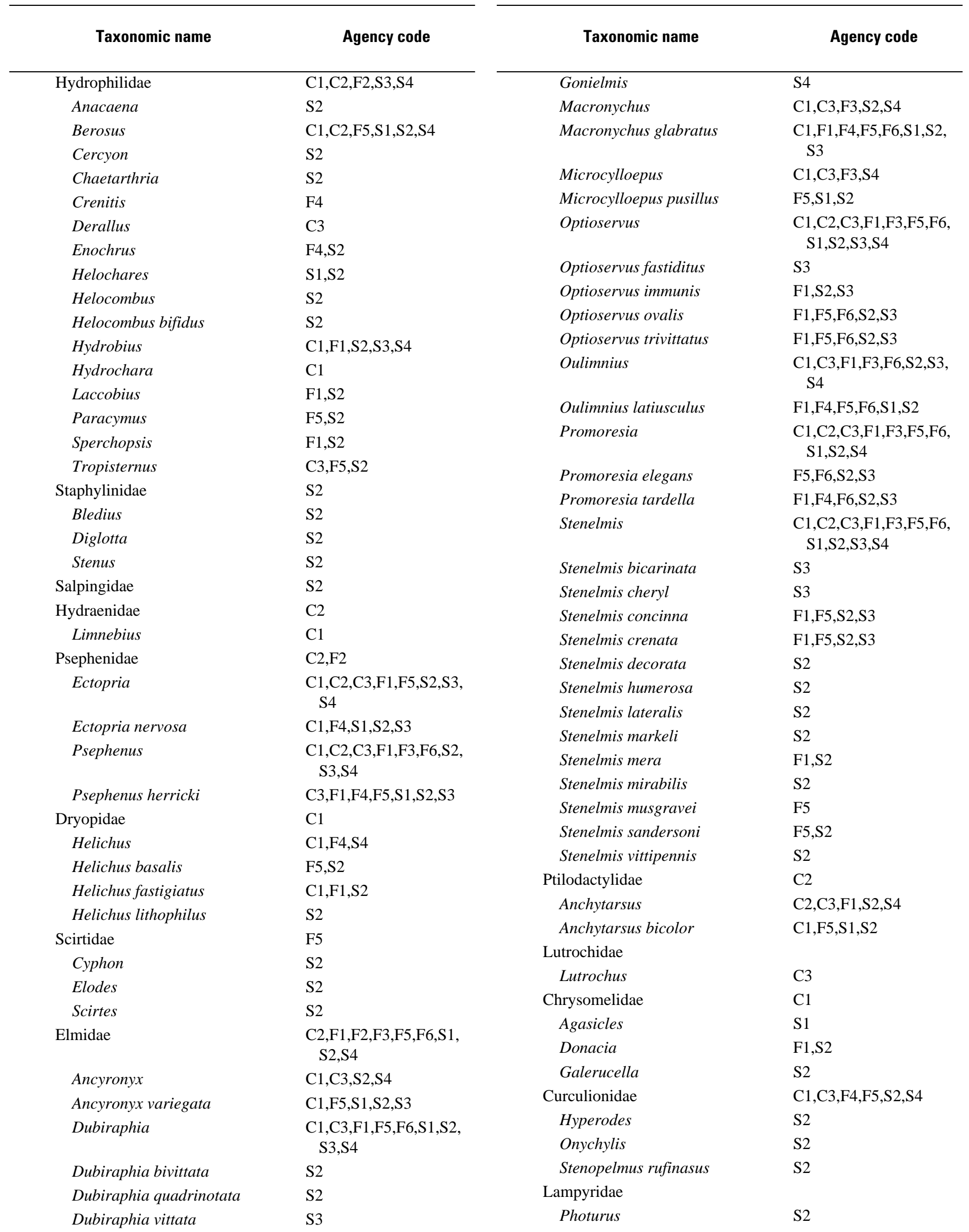




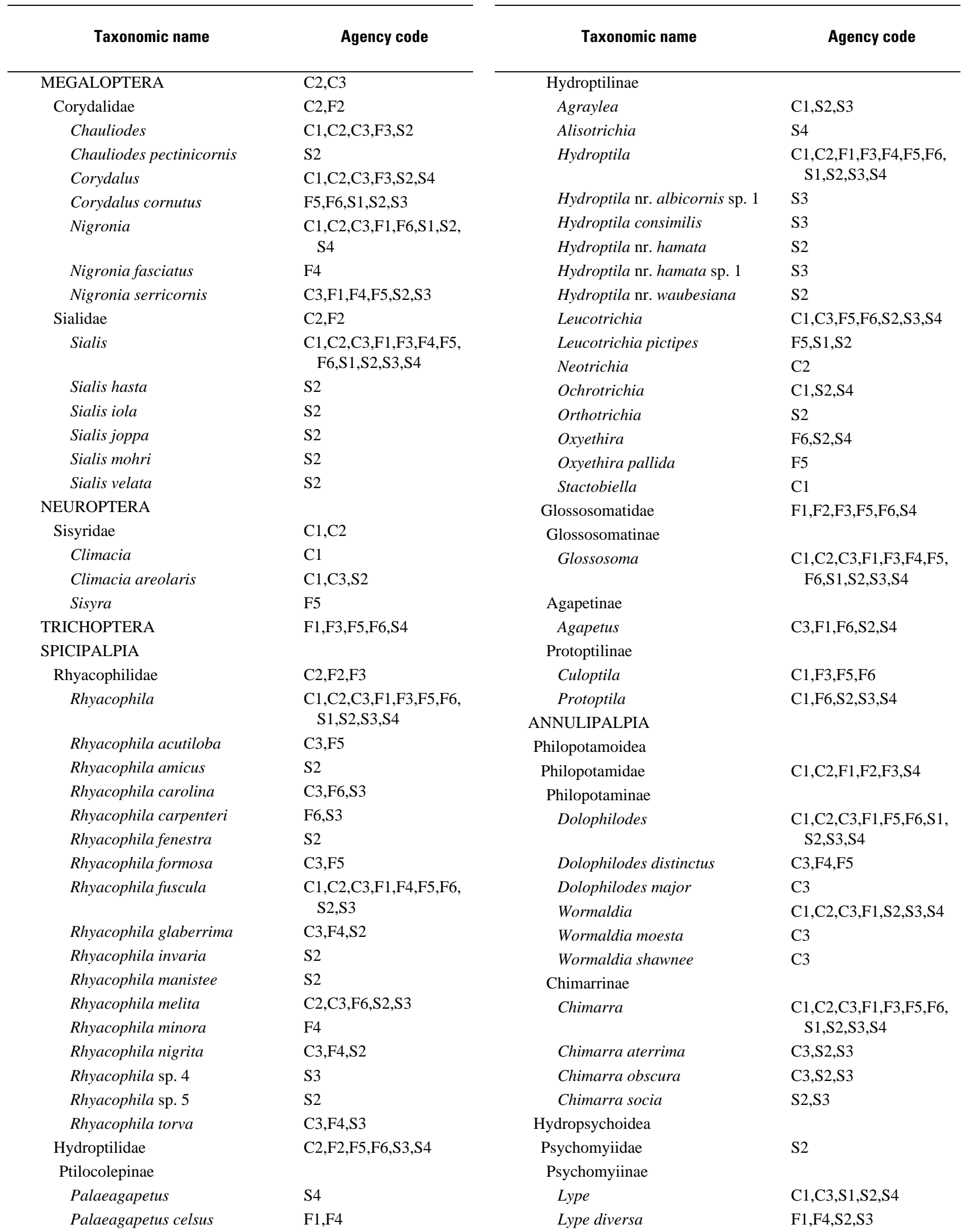




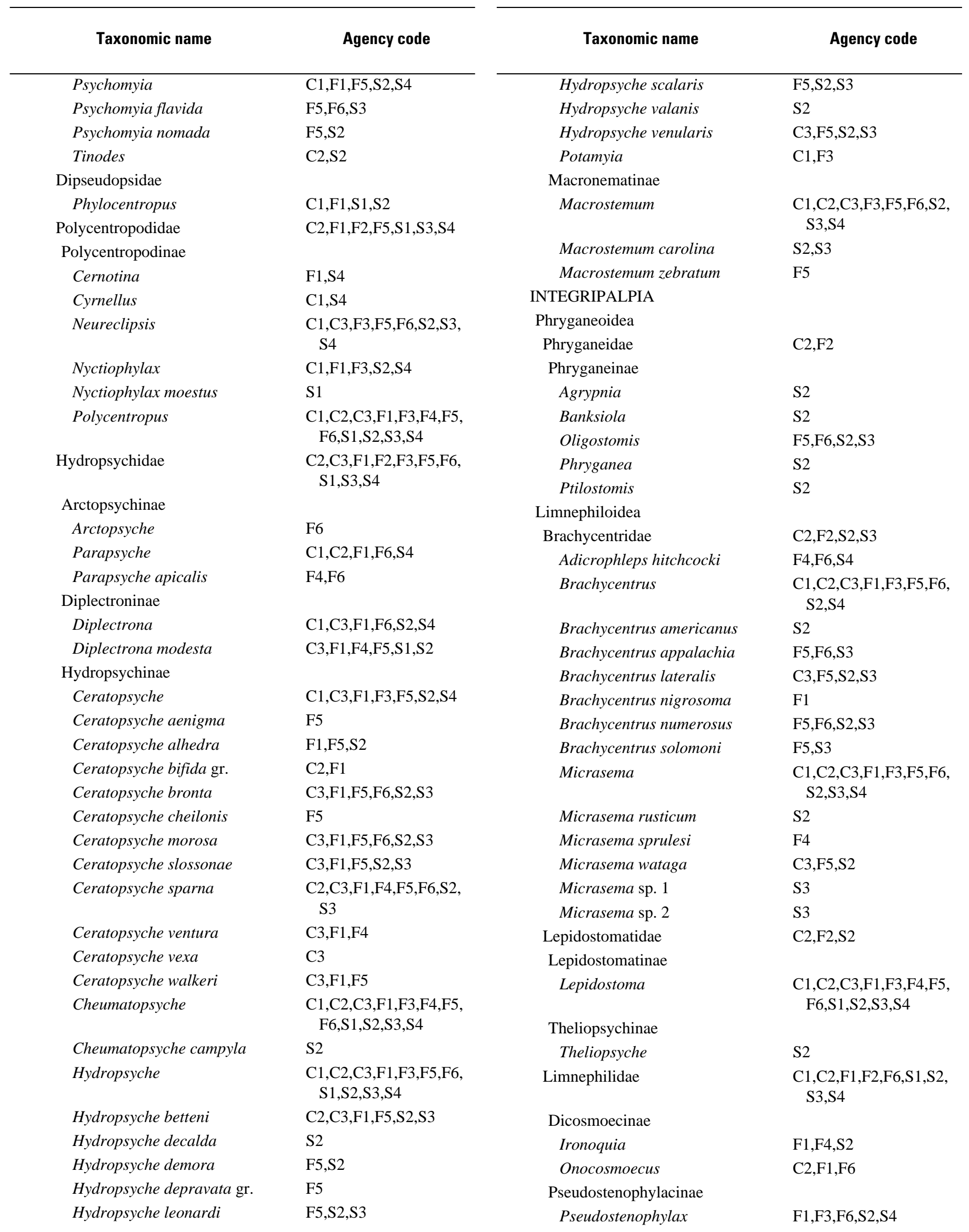




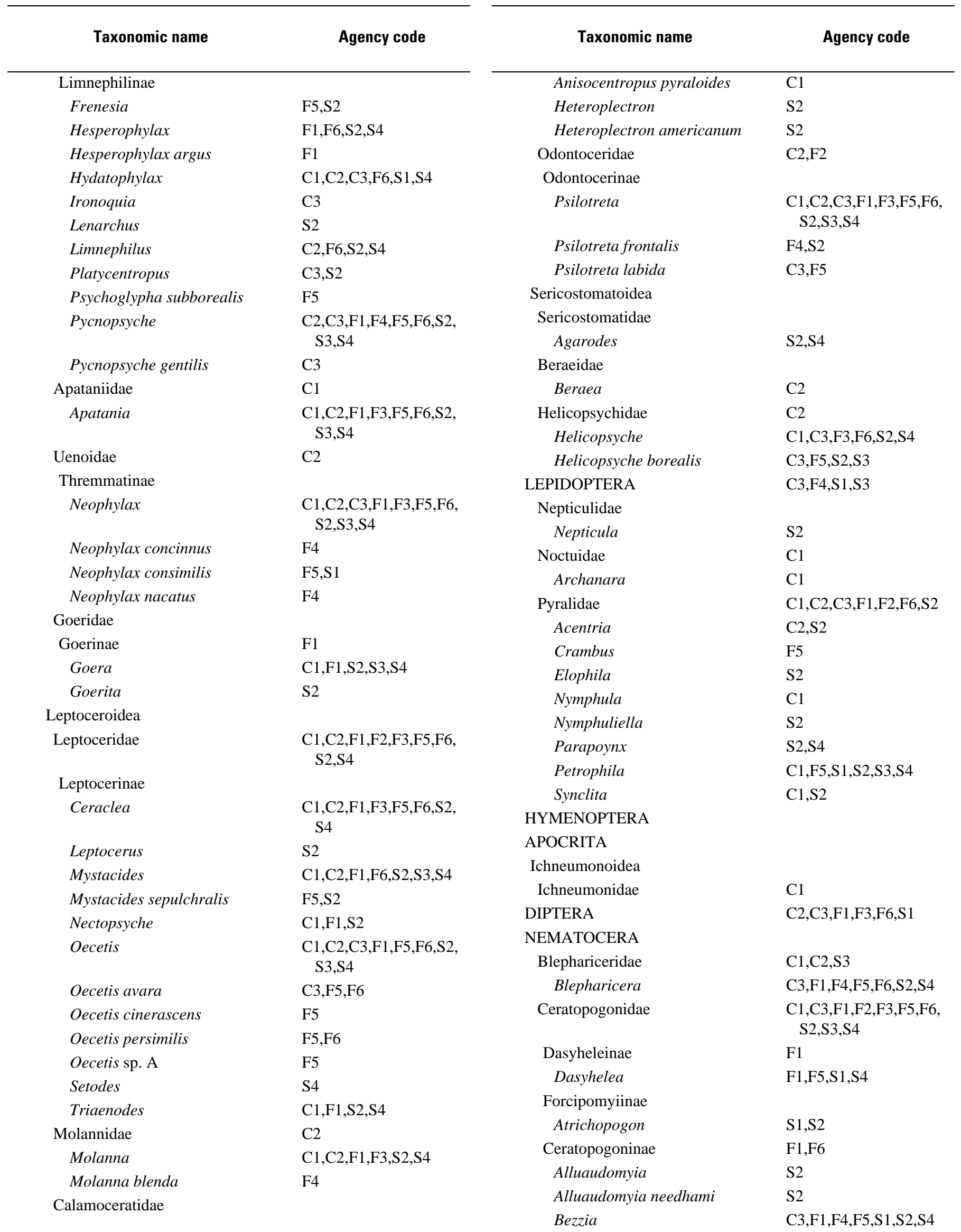




\begin{tabular}{|c|c|c|c|}
\hline Taxonomic name & Agency code & Taxonomic name & Agency code \\
\hline Bezzia glabra & $\mathrm{S} 2$ & Conchapelopia fasciata & S2 \\
\hline Bezzia setulosa & $\mathrm{S} 2$ & Conchapelopia pallens & $\mathrm{S} 2$ \\
\hline Bezzia varicolor & $\mathrm{S} 2$ & Conchapelopia rurika & $\mathrm{S} 2$ \\
\hline Culicoides & $\mathrm{S} 2, \mathrm{~S} 4$ & Hayesomyia & $\mathrm{F} 1$ \\
\hline Culicoides sangusuigus & $\mathrm{S} 2$ & Hayesomyia senata & $\mathrm{S} 2$ \\
\hline Palpomyia & $\mathrm{F} 4, \mathrm{~F} 5, \mathrm{~S} 2$ & Helopelopia & $\mathrm{F} 1$ \\
\hline Palpomyia lineata & S2 & Helopelopia cornuticaudata & $\mathrm{S} 2$ \\
\hline Palpomyia tibialis & $\mathrm{S} 2$ & Hudsonimyia karelena & S3 \\
\hline Chaoboridae & & Meropelopia & $\mathrm{F} 1, \mathrm{~F} 4, \mathrm{~S} 1$ \\
\hline Chaoborus & $\mathrm{C} 1, \mathrm{~S} 2, \mathrm{~S} 4$ & Meropelopia americanus & $\mathrm{F} 1$ \\
\hline Chaoborus punctipennis & $\mathrm{S} 2$ & Meropelopia flavifrons & $\mathrm{F} 1$ \\
\hline Chironomidae & $\begin{array}{l}\mathrm{C} 1, \mathrm{C} 2, \mathrm{C} 3, \mathrm{~F} 1, \mathrm{~F} 2, \mathrm{~F} 3, \mathrm{~F} 5, \\
\mathrm{~F} 6, \mathrm{~S} 1, \mathrm{~S} 2, \mathrm{~S} 4\end{array}$ & $\begin{array}{l}\text { Nilotanypus } \\
\text { Nilotanypus fimbriatus }\end{array}$ & $\begin{array}{l}\text { C1,F1,F6,S4 } \\
\text { F1,S2 }\end{array}$ \\
\hline Tanypodinae & $\mathrm{C} 2, \mathrm{C} 3, \mathrm{~F} 1, \mathrm{~F} 5, \mathrm{~F} 6, \mathrm{~S} 2, \mathrm{~S} 4$ & Paramerina & F1,F5 \\
\hline Coelotanypodini & & Pentaneura & $\mathrm{F} 5, \mathrm{~F} 6, \mathrm{~S} 2, \mathrm{~S} 3, \mathrm{~S} 4$ \\
\hline Clinotanypus & $\mathrm{F} 1, \mathrm{~F} 5, \mathrm{~S} 2$ & Pentaneura carnea & S2 \\
\hline Clinotanypus pinguis & $\mathrm{S} 2$ & Pentaneura inconspicua & S3 \\
\hline Coelotanypus tricolor & $\mathrm{S} 2$ & Rheopelopia & $\mathrm{F} 1, \mathrm{~F} 4, \mathrm{~S} 1, \mathrm{~S} 2$ \\
\hline Macropelopia decedens & $\mathrm{S} 2$ & Trissopelopia & $\mathrm{F} 1, \mathrm{~F} 5, \mathrm{~F} 6, \mathrm{~S} 2$ \\
\hline Psectrotanypus & $\mathrm{S} 2$ & Zavrelimyia & $\mathrm{F} 1, \mathrm{~F} 4, \mathrm{~F} 6, \mathrm{~S} 1, \mathrm{~S} 3$ \\
\hline Psectrotanypus dyari & $\mathrm{S} 2$ & Procladiini & \\
\hline Natarsiini & & Djalmabatista & $\mathrm{S} 2$ \\
\hline Natarsia & $\mathrm{F} 1, \mathrm{~S} 1, \mathrm{~S} 2$ & Procladius & $\mathrm{C} 1, \mathrm{~F} 1, \mathrm{~F} 5, \mathrm{~F} 6, \mathrm{~S} 2, \mathrm{~S} 4$ \\
\hline Natarsia baltimoreus & $\mathrm{F} 1, \mathrm{~F} 4$ & Procladius (Psilotanypus) bellus & $\mathrm{F} 1, \mathrm{~S} 2$ \\
\hline Natarsia fastuosa & $\mathrm{S} 2$ & Procladius culiciformis & $\mathrm{S} 2$ \\
\hline Pentaneurini & & Procladius curtus & $\mathrm{S} 2$ \\
\hline Ablabesmyia & $\mathrm{C} 1, \mathrm{~F} 1, \mathrm{~F} 5, \mathrm{~F} 6, \mathrm{~S} 1, \mathrm{~S} 2$ & Procladius riparius & $\mathrm{S} 2$ \\
\hline Ablabesmyia annulata & $\mathrm{S} 2$ & Tanypodini & \\
\hline Ablabesmyia aspera & $\mathrm{S} 2$ & Tanypus & $\mathrm{F} 1, \mathrm{~S} 2$ \\
\hline Ablabesmyia janta & $\mathrm{S} 2$ & Tanypus neopunctipennis & $\mathrm{S} 2$ \\
\hline Ablabesmyia mallochi & $\mathrm{F} 1, \mathrm{~S} 2$ & Tanypus punctipennis & $\mathrm{S} 2$ \\
\hline Ablabesmyia monilis & $\mathrm{S} 2$ & Tanypus stellatus & $\mathrm{S} 2$ \\
\hline Ablabesmyia peleensis & $\mathrm{S} 2$ & Diamesinae & F5 \\
\hline Ablabesmyia simpsoni & $\mathrm{S} 2$ & Diamesini & \\
\hline Cantopelopia aleta & $\mathrm{S} 2$ & Diamesa & $\mathrm{C} 1, \mathrm{~F} 1, \mathrm{~F} 4, \mathrm{~F} 5, \mathrm{~F} 6, \mathrm{~S} 1, \mathrm{~S} 2$, \\
\hline Conchapelopia & $\mathrm{C} 1, \mathrm{~F} 1, \mathrm{~F} 4, \mathrm{~F} 6, \mathrm{~S} 1, \mathrm{~S} 2$ & & S3,S4 \\
\hline Conchapelopia americana & $\mathrm{F} 1, \mathrm{~S} 2$ & Diamesa nivoriunda & S2 \\
\hline Conchapelopia currani & $\mathrm{S} 2$ & Pagastia & F1,F5,F6 \\
\hline
\end{tabular}




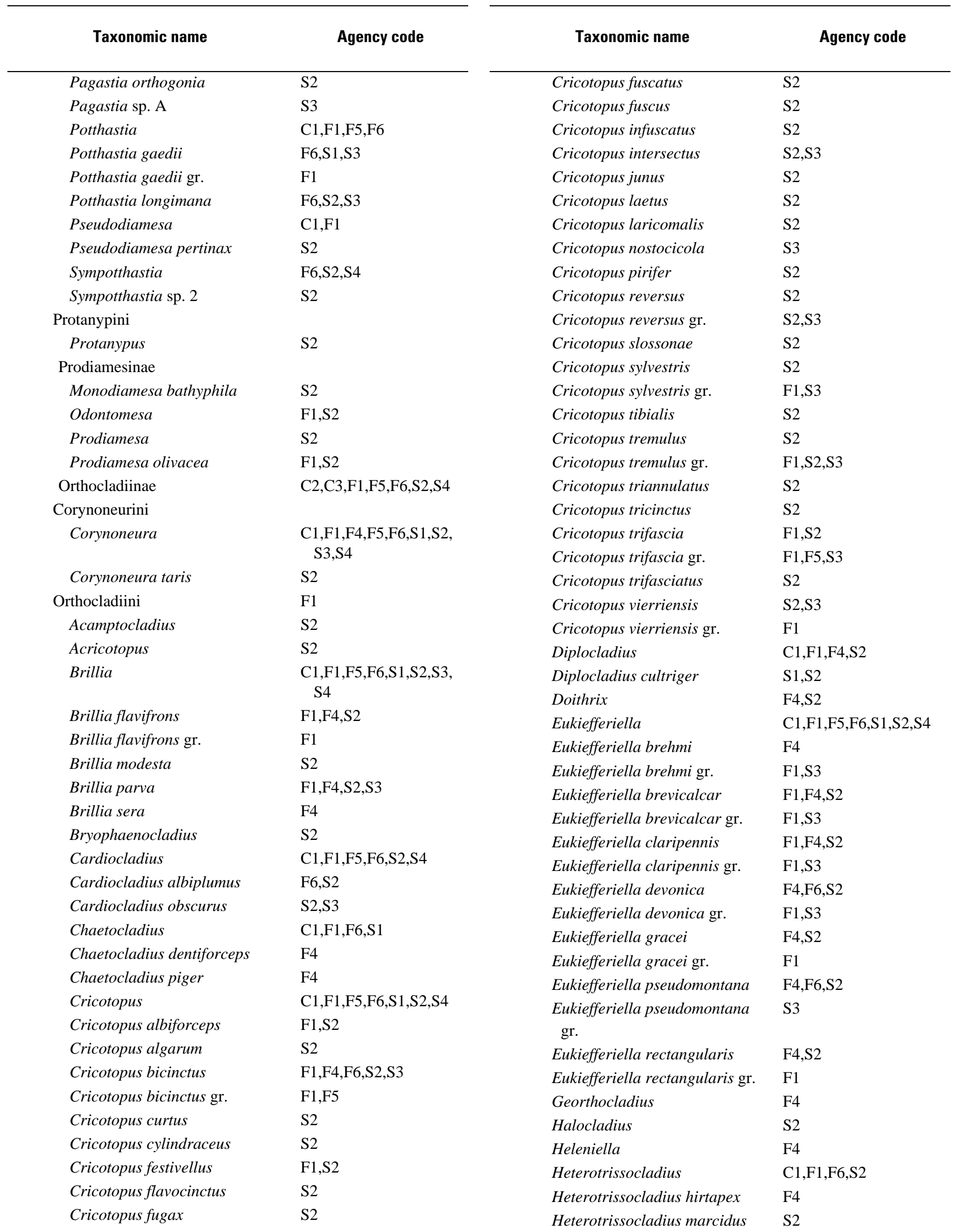




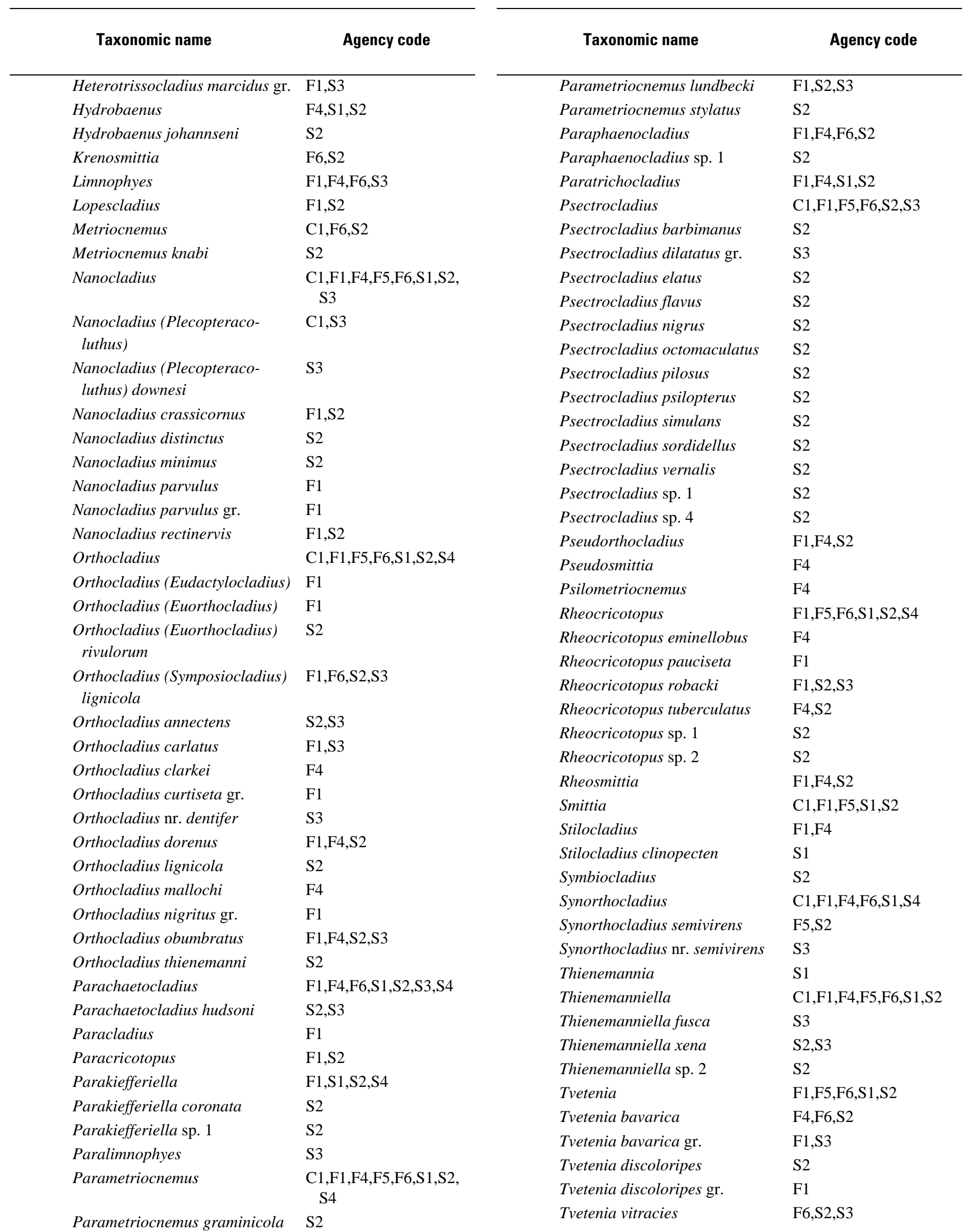


Taxonomic name

$\begin{array}{ll}\text { Unniella } & \mathrm{S} \\ \text { Unniella multivirga } & \mathrm{S} \\ \text { Xylotopus } & \mathrm{S} \\ \text { Xylotopus par } & \mathrm{F} \\ \text { Zalutschia } & \mathrm{S} \\ \text { Zalutschia zalutschicola } & \mathrm{S}\end{array}$

Chironominae

Chironomini

Chironomus

Chironomus decorus

Chironomus riparius

Chironomus tentans

Cladopelma

Cladopelma amachaerus

Cryptochironomus

Cryptochironomus argus

Cryptochironomus fulvus

Cryptochironomus fulvus gr.

Cryptochironomus sp. 1

Cryptochironomus sp. 3

Cryptotendipes

Cryptotendipes emorsus

Cryptotendipes pseudotener

Demicryptochironomus

Demicryptochironomus vulnera-

tus

Demicryptochironomus sp. 3

Dicrotendipes

Dicrotendipes fumidus

Dicrotendipes modestus

Dicrotendipes neomodestus

Dicrotendipes nervosus

Einfeldia

Einfeldia natchitocheae

Endochironomus

Endochironomus nigricans

Endochironomus subtendens

Glyptotendipes

Glyptotendipes lobiferus

Glyptotendipes senilis

Goeldichironomus

Harnischia

Harnischia curtilamellata

Harnischia sp. 2

Hyporhygma

Kiefferulus

Lauterborniella

Lauterborniella agrayloides

Lauterborniella varipennis
Agency code

S2,S3
S2
S2
F1,S2
S2
S2

C2,C3,F1,F5,F6,S4

$\mathrm{C} 3, \mathrm{~F} 1, \mathrm{~F} 5, \mathrm{~F} 6, \mathrm{~S} 4$

$\mathrm{C} 1, \mathrm{~F} 1, \mathrm{~F} 5, \mathrm{~F} 6, \mathrm{~S} 2, \mathrm{~S} 3, \mathrm{~S} 4$

$\mathrm{S} 2$

$\mathrm{S} 2$

$\mathrm{S} 2$

$\mathrm{F} 5, \mathrm{~F} 6, \mathrm{~S} 2$

$\mathrm{S} 2$

$\mathrm{C} 1, \mathrm{~F} 1, \mathrm{~F} 5, \mathrm{~F} 6, \mathrm{~S} 1, \mathrm{~S} 2, \mathrm{~S} 4$

$\mathrm{S} 2$

$\mathrm{S} 2$

S3

S2

S2

$\mathrm{F} 1, \mathrm{~F} 5, \mathrm{~S} 2$

$\mathrm{S} 2$

$\mathrm{S} 2$

$\mathrm{C} 3, \mathrm{~F} 1, \mathrm{~F} 6$

$\mathrm{S} 2$

S3

C1,F1,F5,F6,S1,S2,S4

$\mathrm{F} 1, \mathrm{~S} 2$

$\mathrm{S} 2$

$\mathrm{F} 1, \mathrm{~S} 2, \mathrm{~S} 3$

S2

$\mathrm{F} 1, \mathrm{~S} 2$

S2

$\mathrm{C} 1, \mathrm{~F} 5, \mathrm{~S} 2$

$\mathrm{S} 2$

$\mathrm{S} 2, \mathrm{~S} 3$

$\mathrm{C} 1, \mathrm{~F} 5, \mathrm{~S} 1, \mathrm{~S} 2, \mathrm{~S} 4$

$\mathrm{S} 2, \mathrm{~S} 3$

$\mathrm{S} 2$

$\mathrm{C} 1$

S2

$\mathrm{S} 2$

S2

$\mathrm{S} 2$

$\mathrm{C} 1, \mathrm{~S} 2$

F1

F1,S2

$\mathrm{S} 2$

Taxonomic name

Agency code

Microtendipes

Microtendipes caducus

Microtendipes pedellus

Microtendipes pedellus gr.

Microtendipes rydalensis

Microtendipes rydalensis gr.

Microtendipes tarsalis

Nilothauma

Nilothauma babiyi

Pagastiella

Pagastiella ostansa

Parachironomus

Parachironomus abortivus

Parachironomus frequens

Paracladopelma

Paralauterborniella nigrohal-

terale

Paratendipes

Paratendipes albimanus

Phaenopsectra

Phaenopsectra dyari

Phaenopsectra flavipes

Phaenopsectra obediens

Phaenopsectra obediens gr.

Phaenopsectra punctipes gr.

Phaenopsectra sp. 1

Phaenopsectra sp. 2

Polypedilum

Polypedilum angulum

Polypedilum aviceps

Polypedilum convictum gr.

Polypedilum digitifer

Polypedilum fallax

Polypedilum flavum

Polypedilum halterale

Polypedilum halterale gr.

Polypedilum illinoense

Polypedilum laetum

Polypedilum ontario

Polypedilum ophioides

Polypedilum scalaenum

Polypedilum scalaenum gr.

Polypedilum tritum

Polypedilum sp. C

Robackia

Robackia demeijerei

Robackia demeijerei gr.

Saetheria

Saetheria tylus
C1,F1,F5,F6,S2,S4

$\mathrm{S} 2$

$\mathrm{F} 4, \mathrm{~F} 6, \mathrm{~S} 1, \mathrm{~S} 2$

$\mathrm{F} 1, \mathrm{~S} 3$

F6

F1,S3

S2

$\mathrm{C} 1, \mathrm{~F} 1, \mathrm{~F} 6, \mathrm{~S} 3, \mathrm{~S} 4$

S2

F1,F6

S2

$\mathrm{C} 1, \mathrm{~F} 5, \mathrm{~S} 2$

$\mathrm{S} 2$

S2

$\mathrm{C} 1, \mathrm{~F} 1, \mathrm{~F} 5, \mathrm{~F} 6$

$\mathrm{F} 1, \mathrm{~F} 5, \mathrm{~S} 2$

F1,F4,F5,F6,S1,S2,S4

S2

C1,F1,F5,F6,S1,S2

$\mathrm{F} 1, \mathrm{~S} 3$

$\mathrm{S} 2$

S2

F1

F1

S2

S2

C1,F1,F5,F6,S1,S2,S4

F1

$\mathrm{F} 1, \mathrm{~F} 4, \mathrm{~S} 3$

F1

S3

$\mathrm{F} 1, \mathrm{~S} 2$

$\mathrm{S} 2, \mathrm{~S} 3$

S2

F1

$\mathrm{F} 1, \mathrm{~F} 4, \mathrm{~S} 2, \mathrm{~S} 3$

F1,S3

S2

S2

$\mathrm{S} 2$

F1,S3

$\mathrm{F} 1, \mathrm{~F} 4, \mathrm{~S} 2$

F1

F1

F6,S2

F1

F1,S2,S3,S4

$\mathrm{S} 1, \mathrm{~S} 2$ 


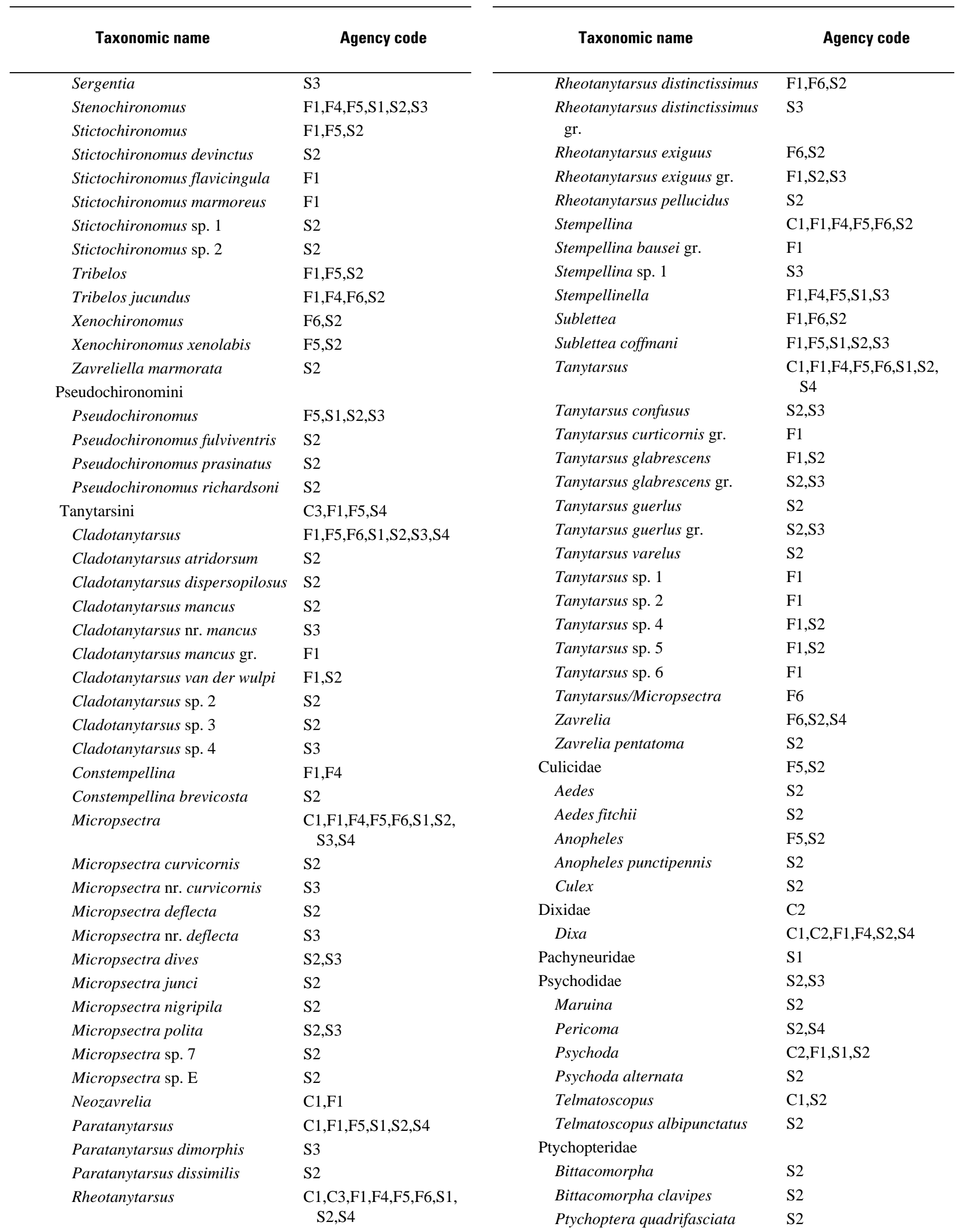




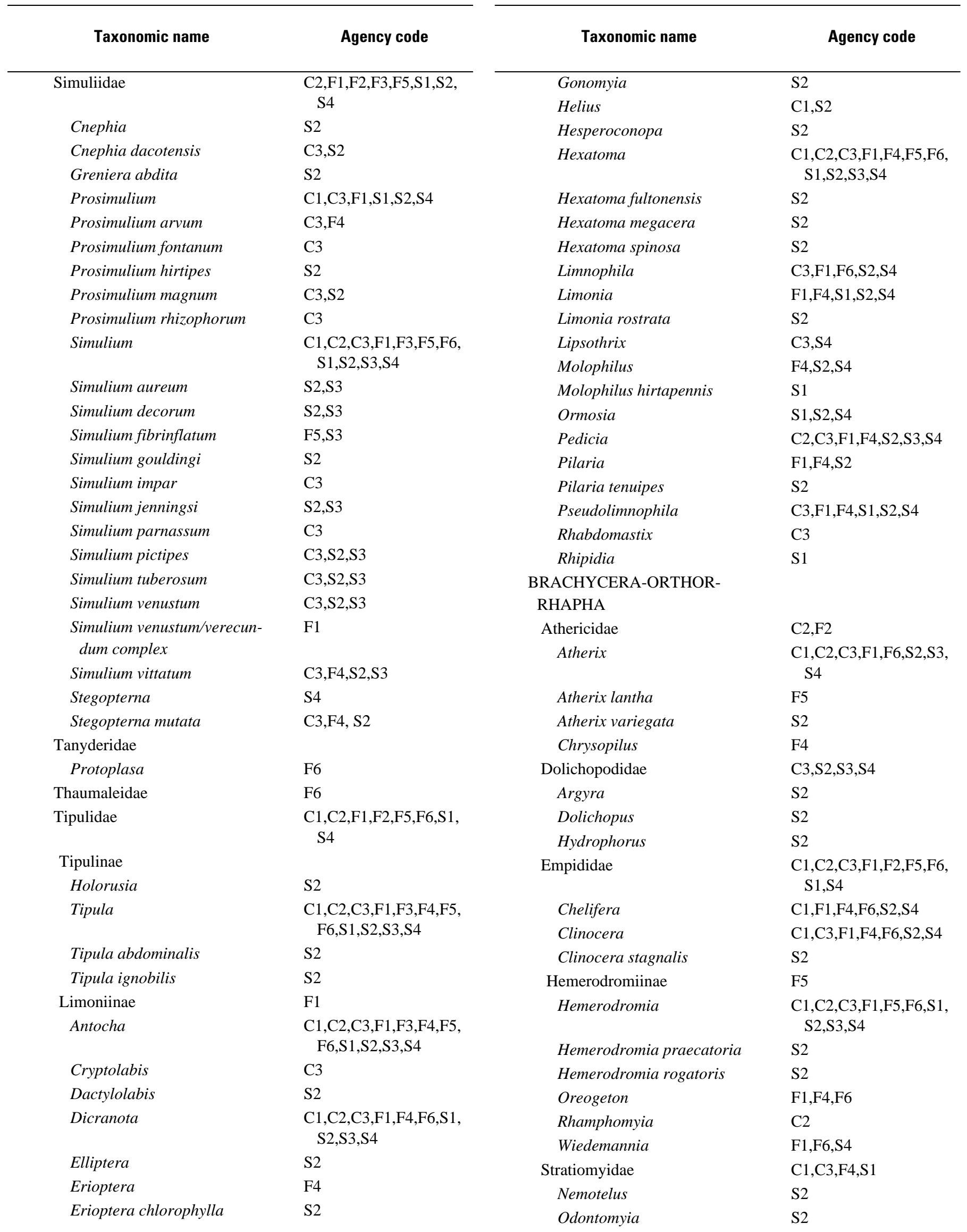


26 A Checklist of the Aquatic Invertebrates of the Delaware River Basin, 1990-2000

\begin{tabular}{|c|c|c|c|}
\hline Taxonomic name & Agency code & Taxonomic name & Agency code \\
\hline Stratiomys & $\mathrm{C} 1, \mathrm{~S} 2$ & Hydrelliinae & \\
\hline Stratiomys discalis & $\mathrm{S} 2$ & Hydrellia & $\mathrm{S} 2$ \\
\hline Tabanidae & $\mathrm{C} 2, \mathrm{~F} 1, \mathrm{~F} 2, \mathrm{~S} 2, \mathrm{~S} 4$ & Muscidae & $\mathrm{C} 1, \mathrm{C} 2, \mathrm{~F} 1$ \\
\hline Chrysops & $\begin{array}{l}\mathrm{C} 2, \mathrm{C} 3, \mathrm{~F} 1, \mathrm{~F} 4, \mathrm{~F} 5, \mathrm{~S} 2, \mathrm{~S} 3 \\
\quad \mathrm{~S} 4\end{array}$ & $\begin{array}{l}\text { Limnophora } \\
\text { Lispe }\end{array}$ & $\begin{array}{l}\mathrm{C} 1, \mathrm{~S} 2 \\
\mathrm{~S} 2\end{array}$ \\
\hline BRACHYCERA-CYCLOR- & & Phoridae & \\
\hline RHAPHA & & Diplonevra & $\mathrm{S} 2$ \\
\hline Ephydridae & $\mathrm{C} 1, \mathrm{C} 2, \mathrm{~F} 6, \mathrm{~S} 2$ & Sciomyzidae & \\
\hline Ephydra & $\mathrm{S} 2$ & Dictya & $\mathrm{S} 2$ \\
\hline Discomyzinae & \multirow{3}{*}{$\mathrm{S} 2$} & Tetanocera & S4 \\
\hline Psilopa & & Syrphidae & $\mathrm{C} 1, \mathrm{~F} 4$ \\
\hline Ephydrinae & & & \\
\hline Brachydeutera & $\mathrm{S} 2$ & & \\
\hline Brachydeutera argentata & $\mathrm{S} 2$ & & \\
\hline Dimecoenia spinosa & $\mathrm{S} 2$ & & \\
\hline Ephydra & $\mathrm{C} 2, \mathrm{~S} 2$ & & \\
\hline Scatella & $\mathrm{S} 2$ & & \\
\hline
\end{tabular}




\section{References Cited}

Adler, P., and Kim, K.C., 1986, The black flies of Pennsylvania (Simuliidae, Diptera) bionomics, taxonomy, and distribution: The Pennsylvania State University, College of Agriculture, Agricultural Experiment Station, University Park, Pa., Bull. 856, 88 p.

Barbour, M.T., Gerrittsen, Jeroen, Snyder, B.D., and Stribling, J.B., 1999, Rapid bioassessment protocols for use in streams and wadeable rivers-Periphyton, benthic macroinvertebrates and fish (2nd ed.): Washington, D.C., U.S. Environmental Protection Agency, Office of Water, EPA 841-B-99002, variously paged.

Bode, R.W., Novak, M.A., and Abele, L.E., 1993a, Biological stream assessment, Willowemoc Creek, 1993 survey: New York State Department of Environmental Conservation Technical Report, 40 p.

Bode, R.W., Novak, M.A., and Abele, L.E., 1993b, Biological stream assessment, Beaver Kill, 1993 survey: New York State Department of Environmental Conservation Technical Report, 34 p.

Bode, R.W., Novak, M.A., and Abele, L.E., 1995a, Biological stream assessment, Willowemoc Creek, 1994 survey: New York State Department of Environmental Conservation Technical Report, 34 p.

Bode, R.W., Novak, M.A., and Abele, L.E., 1995b, Biological stream assessment, Little Beaver Kill, 1994 survey: New York Department of Environmental Conservation Technical Report, 35 p.

Bode, R.W., Novak, M.A., and Abele, L.E., 1995c, Biological stream assessment, Vly Creek, 1995 survey: New York State Department of Environmental Conservation Technical Report, $25 \mathrm{p}$.

Bode, R.W., Novak, M.A., Abele, L.E., Heitzman, D.L., and Smith, A.J., 2004, Thirty-year trends in water quality of rivers and streams in New York state based on macroinvertebrate data 1972-2002: New York State Department of Environmental Conservation Report, 384 p.

Cairns, J., Jr., and Pratt, J.R., 1993, A history of biological monitoring using benthic macroinvertebrates in Rosenberg, D.M., and Resh, V.H., (eds.), Freshwater biomonitoring and benthic macroinvertebrates: New York, N.Y., Chapman and Hall, p. 10-27.

Donnelly, Thomas, 2004a, Distribution of North American Odonata, Part I-Aeshnidae, Petaluridae, Gomphidae, Cordulegastridae: Bulletin of American Odonatology, v. 7, no. 4, p. 61-90.

Donnelly, Thomas, 2004b, Distribution of North American Odonata, Part II-Macromiidae, Corduliidae, and Libellulidae: Bulletin of American Odonatology, v. 8, no. 1, p. 1-32.

Donnelly, Thomas, 2004c, Distribution of North American Odonata, Part III-Calopterygidae, Lestidae, Coenagrionidae, Protoneuridae, Platystictidae, with data sources and bibliography, Parts I-III: Bulletin of American Odonatology, v. 8, no. 2-3, p. 33-99.

Epler, J.H., 1995, Identification manual for the larval Chironomidae (Diptera) of Florida: Orlando, Fla., Florida Department of Environmental Regulation, $302 \mathrm{p}$.

Epler, J.H., 1996, Identification manual for the water beetles of Florida (Coleoptera: Dryopidae, Dytiscidae, Elmidae, Gyrinidae, Haliplidae, Hydraenidae, Hydrophilidae, Noteridae, Psephenidae, Ptilodactylidae, Scirtidae): Tallahassee, Fla., Florida Department of Environmental Protection.

Epler, J.H., 2001, Identification manual for the larval Chironomidae (Diptera) of North and South Carolina.jhepler@concentric.net

Fischer, J.M., Riva-Murray, Karen, Hickman, R.E., Chichester, D.C., Brightbill, R.A., Romanok, K.M., and Bilger, M.D., 2004, Water quality in the Delaware River Basin, Pennsylvania, New Jersey, New York, and Delaware, 1998-2001: U.S. Geological Survey Circular 1227, 38 p.

Hynes, H.B.N.,1960, The biology of polluted waters: Liverpool, England, Liverpool University Press, 199 p.

Hynes, H.B.N., 1970, The biology of running waters: Toronto, Canada, University of Toronto Press, 555 p.

Kathman, R.D., and Brinkhurst, R.O., 1998, Guide to the freshwater oligochaetes of North America: College Grove, Tenn., Aquatic Resources Center, 264 p.

Kennen, J.G., 1999, Relation of macroinvertebrate community impairment to catchment characteristics in New Jersey streams: Journal of the American Water Resources Association, v. 35, no. 4, p. 939-955.

Klemm, D.J., and Lazorchak, J.M., eds., 1994, Environmental and assessment program-Surface water and Region 3 regional environmental monitoring and assessment program 1994_Pilot field operation and methods manual for streams: Cincinnati, Ohio, U.S. Environmental Protection Agency, Environmental Monitoring Systems Lab, Office of Research and Development, EPA/620/R-94/004.

Kolkwitz, R., and Marsson, M., 1908, Okologie der pflanzlichen Saprobien. Berichte der Deutschen Botanischien Gesellschaft 26A: p. 505-519.

Kolkwitz, R., and Marsson, M., 1909. Okologie der tierischen Saprobien. Beitrage zur Lehre von des bioloischen Gewasserbeurteilung. Internationale Revue der Gesamten Hydrobiologie und Hydrographie 2, p. 126-152.

Kosztarab, M., and Schaefer, C.W., eds., 1990, Systematics of North American insects and arachnids-Status and needs: Blacksburg, Va., Information Series 90-1, VA Agric. Exp. Sta., VA Polytechnic Institute and State University.

Kurtenbach, J., 1991, A method for rapid bioassessment of streams in New Jersey using benthic macroinvertebrates: Bulletin of the North American Benthological Society, v. 8, no. 1, p. 129.

Lawrence, G.B., Burns, D.A., Murdoch, P.S., Baldigo, B., and Baevsky, Y.H., 1994, Workplan of the Neversink watershed study in the Catskill Mountains of southeastern New York: U.S. Geological Survey Open-File Report 94-368, $42 \mathrm{p}$. 
Lellis, W.A., 2001, Freshwater mussel survey of the Upper Delaware Scenic Recreational River-Qualitative survey 2000, Report to the National Park Service, February 20,

2001: Wellsboro, Pa., U.S. Geological Survey, 57 p.

Masteller, E.C., Lee, S., Simons, J., and Magee, D., 2004, Searchable database of the caddisflies and stoneflies of Pennsylvania: Pennsylvania Sea Grant, accessed Jan. 31, 2004, at http://www.pserie.psu.edu/seagrant/extension/ other/database.html

Masteller, E.C., and Flint, O.S., Jr., 1992, The Trichoptera (caddisflies) of Pennsylvania-An annotated checklist: Journal of Pennsylvania Academy of Science 66(2): 68-78.

McCafferty, W.P., 2000, Higher classification of the Ephemeroptera: Mayfly Central, Purdue University, Department of Entomology, accessed Jan. 31, 2004, at http://www.entm.purdue.edu/entomology/mayfly/.

Merritt, R.W., and Cummins, K.W., 1996, An introduction to the aquatic insects of North America (3d ed.): Dubuque, Iowa, Kendall/Hunt Publishing Company, 862 p.

Monroe County Planning Commission, 1990, Water quality study, 1990: Stroudsburg, Pa., Monroe County Planning Commission Report, $50 \mathrm{p}$.

Monroe County Planning Commission, 1991, Water quality study, 1991: Stroudsburg, Pa., Monroe County Planning Commission Report, 47 p.

Monroe County Planning Commission. 1992. Water Quality Study, 1992: Stroudsburg, Pa., Monroe County Planning Commission Report, 54 p.

Monroe County Planning Commission, 1993, Water Quality Study, 1993: Stroudsburg, Pa., Monroe County Planning Commission Report, 84 p.

Monroe County Planning Commission, 1994, Water Quality Study, 1994: Stroudsburg, Pa., Monroe County Planning Commission Report, 93 p.

Monroe County Planning Commission, 1995, Water Quality Study, 1995: Stroudsburg, Pa., Monroe County Planning Commission Report, 95 p.

Monroe County Planning Commission, 1996, Water Quality Study, 1996: Stroudsburg, Pa., Monroe County Planning Commission Report, 101 p.

Monroe County Planning Commission, 1997, Water Quality Study, 1997: Stroudsburg, Pa., Monroe County Planning Commission Report, 100 p.

Monroe County Planning Commission, 1998, Water Quality Study, 1998: Stroudsburg, Pa., Monroe County Planning Commission Report, 104 p.

Monroe County Planning Commission, 1999, Water Quality Study, 1999: Stroudsburg, Pa., Monroe County Planning Commission Report, 75 p.

Monroe County Planning Commission, 2000, Water Quality Study, 2000: Stroudsburg, Pa., Monroe County Planning Commission Report, $82 \mathrm{p}$.

New Jersey Department of Environmental Protection, 1994a, Ambient biomonitoring network-Delaware River drainage basin, 1992 benthic macroinvertebrate data: New Jersey Department of Environmental Protection Report, 206 p.
New Jersey Department of Environmental Protection, 1994b, The establishment of ecoregion biological reference sites for New Jersey streams-Incorporating habitat quality and benthic macroinvertebrate communities, 1989-1993 monitoring data: New Jersey Department of Environmental Protection Report, $114 \mathrm{p}$.

New Jersey Department of Environmental Protection. 1996. Ambient biomonitoring network-Lower Delaware River drainage basin, 1995-96 benthic macroinvertebrate data: New Jersey Department of Environmental Protection Report, $133 \mathrm{p}$.

New Jersey Department of Environmental Protection, 1999a, Ambient biomonitoring network-Watershed management areas 1, 2, and 11-Upper Delaware region, 1998 benthic macroinvertebrate data: New Jersey Department of Environmental Protection Report, $171 \mathrm{p}$.

New Jersey Department of Environmental Protection, 1999b, Ambient biomonitoring network-Watershed management areas 19 and 20, Delaware Region, upper tidal portion, 1998 benthic macroinvertebrate data: New Jersey Department of Environmental Protection Report, 113 p.

New York State Department of Environmental Conservation, 1996, The Delaware River Drainage Basin Biennial Report, 1993-94: New York State Department of Environmental Conservation Technical Report, 102 p. + appendixes.

Omernick, J.M., 1987, Ecoregions of the conterminous United States: Annals of the Association of American Geographers v. 77, no. 1, p. 118-125.

Passmore, M., and Green, J., 1997, The development of a stream reference condition in Monroe County, Pennsylvania: U.S. Environmental Protection Agency, Ecological Assessment and Protection Division Report, $61 \mathrm{p}$.

Pike County Conservation District, 1991, Annual Report, surface water quality monitoring program: Hawley, Pa., Pike County Conservation District Report, 81 p.

Pike County Conservation District, 1992, Annual Report, surface water quality monitoring program: Hawley, Pa., Pike County Conservation District Report, 110 p.

Pike County Conservation District, 1993, Annual Report, surface water quality monitoring program: Hawley, Pa., Pike County Conservation District Report, 104 p.

Pike County Conservation District, 1994, Annual Report, surface water quality monitoring program: Hawley, Pa., Pike County Conservation District Report., 117 p.

Pike County Conservation District, 1995, Annual Report, surface water quality monitoring program: Hawley, Pa., Pike County Conservation District Report, $142 \mathrm{p}$.

Pike County Conservation District, 1996, Annual Report, surface water quality monitoring program: Hawley, Pa., Pike County Conservation District Report, 138 p.

Pike County Conservation District, 1997, Annual Report, surface water quality monitoring program: Hawley, Pa., Pike County Conservation District Report.

Pike County Conservation District, 1998, Annual Report, surface water quality monitoring program: Hawley, Pa., Pike County Conservation District Report, 136 p. 
Pike County Conservation District, 1999, Environmental quality of Pike County streams using bioassessment techniques: Hawley, Pa., Pike County Conservation District Report, 55 p.

Pike County Conservation District, 2000, Environmental quality of Pike County streams using bioassessment techniques: Hawley, Pa., Pike County Conservation District Report, $56 \mathrm{p}$.

Plafkin, J.L., Barbour, M.T., Porter, K.D., Gross, S.K., and Hughes, R.M., 1989, Rapid bioassessment protocols for use in streams and rivers-Benthic macroinvertebrates and fish: Washington, D.C., U.S. Environmental Protection Agency, Office of Water, EPA/444/4-89-001, variously paged.

Rawlins, J.E., and Bier, C.W., 1998, Invertebrates-Review of status in Pennsylvania, in Hassinger, J.D., Hill, R.J., Storm, G.L., and Yahner, R.H., eds., Inventory and monitoring of biotic resources in Pennsylvania-Current ecological and landscape topic, Vol. 1., Pennsylvania Biological Survey: University Park, Pa., The Pennsylvania State University, Center for Biodiversity Research, 220 p.

Reif, A.G., 1999, Physical, chemical, and biological data for selected streams in Chester County, Pennsylvania, 198194: U.S. Geological Survey Open-File Report 99-216, $607 \mathrm{p}$.

Reif, A.G., 2000, Physical, chemical, and biological data for selected streams in Chester County, Pennsylvania, 1995-97: U.S. Geological Survey Open-File Report 00-238, 147 p.

Shertzer, R.H., and Schreffler, T.L., 1996, Pennsylvania's surface water quality monitoring network: Harrisburg, Pa., Pennsylvania Department of Environmental Protection, Report No. 3600-BK-DEP0636.

Smith, D.G., 2001, Pennak's freshwater invertebrates of the United States-Porifera to Crustacea: New York, John Wiley and Sons, Inc., 638 p.

Smith, I.M., and Cook, D.R., 1991, Water mites, in Thorp, J.H., and A.P. Covich, A.P., eds., Ecology and classification of North American freshwater invertebrates: San Diego, Calif., Academic Press, Inc., p. 523-592.

Snyder, C., Young, J., Smith, D., Ross, R., and Bennett, R., 1999, Influence of eastern hemlock on aquatic biodiversity in the Delaware Water Gap National Recreation Area:

Kearneysville, W.Va., U.S. Geological Survey, Leetown Science Center.
Stewart, K.W., and Stark, B.P., 2002, Nymphs of North American Stonefly Genera (Plecoptera) (2d ed.): Columbus, Ohio, The Caddis Press, 510 p.

Strayer, D.L., and Jirka, K.J., 1997, The pearly mussels of New York State: New York State Museum Memoir 26: 1-113.

Thorp, J.H., and Covich, A.P., 1991, Ecology and classification of North American freshwater invertebrates: San Diego, Calif., Academic Press, Inc., 911 p.

Thorp, J.H., and Covich, A.P., 2001, Ecology and classification of North American freshwater invertebrates ( $2 \mathrm{~d}$ ed.): San Diego, Calif., Academic Press, Inc., 1056 p.

U.S. Environmental Protection Agency, 1994, Guidance on implementation of biological criteria-Draft: Washington, D.C., U.S. Environmental Protection Agency, Office of Science and Technology.

U.S. Environmental Protection Agency, 1999, Level III Ecoregions of the Conterminous United States (revision of Omernick 1987): Corvallis, Oreg., U.S. Environmental Protection Agency, National Health and Environmental Effects Laboratory, Western Ecology Division.

U.S. Environmental Protection Agency, 2000, Environmental monitoring and Assessment Program EMAP: accessed November 2001 at http://www.epa.gov/emap

Vogelmann, J.E., Sohl, T., and Howard, S.M., 1998, Regional characterization of land cover using multiple sources of data: Photogrammetric Engineering and Remote Sensing, v. 64, no. 1, p. 45-57.

Wetzel, M.J., 2001, The aquatic Annelida occurring in or adjacent to the Great Smokey Mountains National Park-An annotated checklist of species: Champaign, Ill., Illinois Natural History Survey, Center for Biodiversity.

Wiggins, G.B., 1995, Larvae of the North American caddisfly genera (Trichoptera) (2d ed.): Toronto, Canada, University of Toronto Press, $457 \mathrm{p}$.

Wilson, E.O., 1984, Biophila: Cambridge, Mass., Harvard University Press, $111 \mathrm{p}$.

Wilson, E.O., ed., 1988, Biodiversity: Washington, D.C., National Academy Press, 621 p.

Wilson, E.O., 2000, A global biodiversity map: Science, v. 289, p. 2279.

Young, C., and Gelhaus, J., 2000, Crane flies of Pennsylvania-Preliminary checklist and database development with emphasis on aquatic species: Report to the Wild Resources Conservation Fund and the Pennsylvania Fish and Boat Commission, $256 \mathrm{p}$. 